\title{
Transitions of orbifold vacua
}

\author{
Kang-Sin Choi ${ }^{a, b}$ and Tatsuo Kobayashi ${ }^{c}$ \\ ${ }^{a}$ Scranton Honors Program, Ewha Womans University, \\ Seoul 03760, Korea \\ ${ }^{b}$ Department of Physics and Astronomy, University of California, \\ Irvine, California 92697, U.S.A. \\ ${ }^{c}$ Department of Physics, Hokkaido University, \\ Sapporo 060-0810, Japan \\ E-mail: kangsin@ewha.ac.kr, kobayashi@particle.sci.hokudai.ac.jp
}

ABSTRACT: We study the global structure of vacua of heterotic strings compactified on orbifolds $T^{4} / \mathbb{Z}_{N}(N=2,3)$ in the presence of heterotic 5 -branes. Gauge symmetry breaking associated with orbifold is described by instantons in the field theory. There is phase transition between small (zero size limit) instantons and heterotic 5-branes. This mechanism provides top-down, stringy account to the spectrum and modular invariance condition of non-perturbative vacua with 5-branes. Also it takes us from one vacuum to another by emitting and absorbing instantons. This means that many vacua with different gauge theories are in fact connected and are inherited from perturbative vacua. It follows that there is also transition of twisted instantons at the fixed points. Thus we can understand transitions among perturbative vacua as well.

Keywords: Superstring Vacua, Brane Dynamics in Gauge Theories, Superstrings and Heterotic Strings

ArXIV EPRINT: 1901.11194 


\section{Contents}

1 Introduction 1

1.1 Global consistency condition 2

1.2 Small instantons 4

2 Orbifold vacua 5

2.1 Perturbative spectrum 5

2.2 Perturbative modular invariance 5

2.3 Classification of perturbative vacua $\quad 6$

2.4 Instantons on $\mathbb{R}^{4} / \mathbb{Z}_{N}$ and $T^{4} / \mathbb{Z}_{N}$ orbifold 8

$\begin{array}{ll}2.5 & \text { Chirality of localized fields } \\ \end{array}$

3 Non-perturbative vacua and their transitions $\quad \mathbf{1 2}$

$\begin{array}{ll}3.1 \text { Small instantons into heterotic 5-branes } & 12\end{array}$

$\begin{array}{lll}3.2 & \text { Modified modular invariance condition } & 13\end{array}$

3.3 Example 14

$\begin{array}{ll}3.4 & \text { Recombination of spectrum and anomaly flow } \\ & 16\end{array}$

$\begin{array}{ll}3.5 & \text { Dual and inverse transitions } \\ \end{array}$

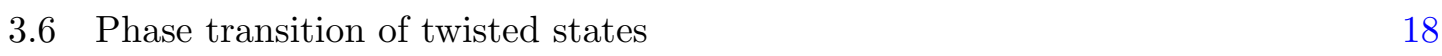

$\begin{array}{lll}3.7 & \text { Selection rule } & 20\end{array}$

4 Connected vacua $\quad 22$

4.1 Connected vacua of $\mathbb{Z}_{3}$ orbifold 22

4.2 Communication between two $E_{8}$ 's 28

5 Discussion $\quad 29$

\section{Introduction}

Well-known is phase transition of small instantons, by which instantons may shrink to zero size and become heterotic 5-branes [1-3]. It recently attracted renewed attention in various contexts. For instance, geometry of F-theory helps us identify non-perturbative states and describe the phase transitions [4-10]. Such transition is important in understanding six dimensional superconformal field theory (SCFT) which is based on dynamics of M5-branes, dual to these heterotic 5-branes. In particular, the mysterious non-Abelian nature of such SCFT is associated with the dynamics of M5-branes. It is also tightly associated to the dynamics of non-critical strings, describing fluctuation of M5-branes, which can be studied by string theory.

In this paper, we use this phase transition to study vacua of heterotic strings, $E_{8} \times E_{8}$ and $\mathrm{SO}(32)$, compactified on toroidal orbifolds $T^{4} / \mathbb{Z}_{N}, N=2,3$ [11-20]. This provides a 
new top-down way to study vacua of orbifold compactification in the presence of heterotic 5-branes, opening more possibilities for realistic vacua for the Standard Model. It also leads us to understand global structure of moduli space.

Instantons on non-compact orbifold $\mathbb{R}^{4} / \mathbb{Z}_{N}$, which approximates a local geometry at the orbifold fixed points of $T^{4} / \mathbb{Z}_{N}$, provides a flat (constant) background connection at infinity $[13,17,21]$. This information is parameterized by so-called shift vector $V$, which enters as an input in the string worldsheet CFT. It is an outstanding observation in ref. [17] that the spectrum in the presence of heterotic 5-branes can be still be calculated using the same CFT just by modifying the zero point energy, proportional to the number of heterotic 5-branes. However there have been bottom-up approaches, in which quantitative factors are empirically determined and anomaly cancellation was used for consistency condition. In this paper we seek top-down approach, enabling justification on the calculation formulae and systematic classification of vacua.

In the heterotic orbifold theories, the worldsheet CFT is exactly solvable [11, 12] so that we can track the change of spectrum. This solvability includes the spectrum in twisted sectors in which the fields are localized at the orbifold fixed points, which are singular and cannot be accessed by field theory. In stringy calculation a shift vector $V$ completely determines the twisted field spectrum. Roughly speaking, using transition from perturbative orbifold vacua to non-perturvative ones, we can extract the information about small instantons from shift vectors by decomposing it, $V=V_{1}+V_{2}$ into still broken part $V_{1}$ and instanton part $V_{2}$. The non-perturbative model is described by the new shift vector $V_{1}$. Thus we may hope that the above decomposition is possible and we can explain the non-perturbative vacua in terms of perturbative ones.

An interesting consequence is that many different vacua, described by different shift vectors or instanton data, can be connected. Chains of phase transitions enable us to move from one vacuum to another. Whether given string vacua are connected is an important problem. For instance, we may understand to what extent the Standard Model is unique. By symmetry breaking and energy consideration, we can seek a mechanism for dynamical selection of vacua.

\subsection{Global consistency condition}

To obtain realistic vacua, we break the symmetry of heterotic string by associating it with the symmetry of internal space. In orbifold compactification, the information on the gauge symmetry is parameterized by a shift vector and we obtain spectrum by a projection associated with it that we review shortly.

The low energy limit of heterotic string is described by supergravity. In the smooth compactification, the supersymmetry preserving solution for the gauge field is the semistable vector bundle [23]. In six dimensions, such vector bundle is a set of instantons [24]. The gauge symmetry is broken by this instanton background: if the structure group of the instantons is $G$, then that unbroken group is the commutant $H$ of $G$ in the mother group of heterotic string, $E_{8} \times E_{8}$ or $\operatorname{Spin}(32) / \mathbb{Z}_{2}$ (which we usually call $\mathrm{SO}(32)$ for convenience). What the shift vector describes should be related to this instanton background. It is also subject to the following global consistency condition. 
The global consistency condition is given by the Bianchi identity for the rank two antisymmetric $B$-field

$$
d H=d^{2} B+\frac{1}{8 \pi^{2}} \operatorname{tr} R^{2}-\frac{1}{8 \pi^{2}} \operatorname{Tr} F^{2}=0 .
$$

Here $\mathrm{Tr}$ is the trace over adjoint representation of the gauge group, normalized by the corresponding dual Coxeter number. Also $R$ and $F$ are curvature two forms for the Lorentz and gauge connections, respectively, and a shorthand notation for the wedge product is used, e.g. $F^{n} \equiv \wedge^{n} F$. We note that the curvatures provide the magnetic source to the $B$-field.

If we take a background such that $d^{2} \bar{B}=0$, the last term in (1.1) under the instanton background $\operatorname{Tr} \bar{F}^{2} /\left(8 \pi^{2}\right)$ is, integrated over a compact manifold, integrally quantized instanton number $k$. The orbifold geometry determines the second term $\operatorname{tr} \bar{R}^{2} /\left(8 \pi^{2}\right)$ and is integrated to give 24, the Euler number of the K3. Thus we have

$$
24-k=0 .
$$

For $E_{8} \times E_{8}$ heterotic string, the contribution on the gauge field can be decomposed into that of each $E_{8}$ and gives $k=k_{1}+k_{2}$, with obvious notations.

We can regard a $\mathrm{K} 3$ as a blown-up $T^{4} / \mathbb{Z}_{N}$ orbifold, whose details we will study in section 2.4. In the orbifold limit, there appear localized fields at the orbifold fixed points. We will also see that the instanton number in (1.1) can be decomposed into the bulk contribution $\bar{F}_{\mathrm{U}}$ and the fixed point contribution $\bar{F}_{\mathrm{T}}$. The total sum should be preserved so that

$$
\frac{1}{8 \pi^{2}} \operatorname{tr} \bar{R}^{2}-\frac{1}{8 \pi^{2}} \operatorname{Tr} \bar{F}_{\mathrm{U}}^{2}-\frac{1}{8 \pi^{2}} \operatorname{Tr} \bar{F}_{\mathrm{T}}^{2}=0 .
$$

Integrating over the entire manifold, the relation (1.3) is translated into the relations among numbers

$$
24-k_{\mathrm{U}}-k_{\mathrm{T}}=0 .
$$

We may call the object contributing to $\operatorname{Tr} \bar{F}_{\mathrm{T}}^{2}$ a twisted instanton.

Now we introduce a heterotic 5 -brane. It provides magnetic source to $B$-field. Supposing that they fill in the six dimensional uncompact space and look point-like in the internal space, so they satisfy the equation of motion

$$
d H=\sum_{a} \delta^{(4)}\left(x-x_{a}\right)
$$

which is Bianchi identity (1.1) modified to [10, 25, 26]

$$
\frac{1}{8 \pi^{2}} \operatorname{tr} \bar{R}^{2}-\sum_{a=1}^{n} \delta^{(4)}\left(x-x_{a}\right)-\frac{1}{8 \pi^{2}} \operatorname{Tr} \bar{F}^{2}=0,
$$

where $a=1, \ldots, n$ labels different heterotic 5-branes. Integrating this over the entire orbifold, we obtain a consistency condition [10, 25]

$$
24-n-k=0 \text {. }
$$


Regarding (1.7) as a conserved invariant, we may ask whether there can be transfer between the two numbers $n$ and $k$. As will see shortly, if instantons shrink to zero size so that the curvature $\operatorname{Tr} \bar{F}^{2}$ becomes $\delta$-function, the corresponding contribution of $k$ then contributes to $n$. This is not possible for the Riemann curvature because the corresponding term has the opposite sign to the $\delta$-function.

In this paper, we make extensive use of this phase transition to study the global structure of orbifold vacua.

\subsection{Small instantons}

An instanton, which we need for the reason discussed above, has continuous size. It may shrink to zero size becoming 'small instanton.' Although the resulting profile becomes singular, the broken group, identical to structure group $G$, is restored $[1,4,5]$. Small instantons are codimension four and undergo phase transition into heterotic 5-branes [27]. The behavior is slightly different in two heterotic string theories.

In the $\mathrm{SO}(32)$ heterotic string, there emerges extra gauge group $\mathrm{Sp}(1) \simeq \mathrm{SU}(2)$ for each small instanton. We have also a hypermultiplet transforming as a bifundamental under $H \times \mathrm{SU}(2)$; the multiplicity is $\frac{1}{2}$ due to half representation. This can be easily understood in type I dual, in which the spacetime of the $\mathrm{SO}(32)$ theory and heterotic 5branes are respectively mapped to D9/O9 and D5 branes. Each heterotic 5-brane gives rise to $\mathrm{Sp}(1)$ gauge group due to the projection from $\mathrm{O} 9$ and when $n$ heterotic 5 -branes become all coincident it is enhanced to $\operatorname{Sp}(n)$. At the intersection between D9/O9 and D5, we have the bifundamental representation under $\mathrm{SO}(32) \times \mathrm{Sp}(n)$ group or its subgroup, from an open string connecting between them. Six dimensional gauge anomaly is nontrivial and it provides an important clue in understanding its non-perturbative structure.

In the $E_{8} \times E_{8}$ string, there is no additional gauge group from the 5-branes, but there is another interesting new effect. We can go to strong coupling limit to have M-theory with a new dimension $[4,5,28,29]$. Two $E_{8}$ 's are separately localized at the end of the interval, which are interpreted as another 9-branes. There can be a tensor branch in which such heterotic 5-branes are pulled out into the bulk and become M5-branes [3].

In what follows, we will apply this phenomenon to strings on toroidal orbifold $T^{4} / \mathbb{Z}_{N}$, formed by a discrete rotational element of $\mathbb{Z}_{N} \subset \mathrm{SU}(2)$ holonomy on the torus. They can be regarded as a singular limit of the K3 manifold. In the smooth limit, instantons are spread over the internal manifold, as vector bundles. When we have orbifold limit, embedded instantons are localized at the fixed points. Their data are usually described by a shift vector $V$, which is also explained by instantons in refs. [19, 21, 30, 31], where the transition between smooth and singular manifold is described. In this limit the structure group is the Cartan subalgebra corresponding to non-vanishing components of $V$.

In the previous works, emphases have been made on the effect of heterotic 5-branes or instantons separately and the phase transition was not applied. In this paper we apply the phase transition idea to understand the connection between them. When there is a phase transition making small instantons into heterotic 5-branes we can track the change in the shift vector. This provides a top-down approach to understand the effect of nonperturbative effect. 


\section{Orbifold vacua}

First we review perturbative orbifold vacua. The stringy spectrum is obtained by CFT with orbifold projection guided by modular invariance. However we can gain physical understanding on what is happening in terms of instantons.

\subsection{Perturbative spectrum}

We compactify heterotic string on toroidal orbifold $T^{4} / \mathbb{Z}_{N}$ to yield six non-compact dimensions. We are particularly interested in prime cases $N=2,3$. With the complexified coordinates $z^{1}=x^{6}+e^{2 \pi i / 3} x^{7}, z^{2}=x^{8}+e^{2 \pi i / 3} x^{9}$ in the torus, the order $N$ twist acts as

$$
\left(z^{1}, z^{2}\right) \rightarrow\left(e^{2 \pi i \phi_{1}} z^{1}, e^{2 \pi i \phi_{2}} z^{2}\right), \quad \phi=\left(\frac{1}{N},-\frac{1}{N}\right) .
$$

This preserves half of the supersymmetry.

The current algebra is described by the weight vector $P$. We associate this rotation with a shift of the weight vector $P \rightarrow P+V$, where $V$ is the order $N$ shift vector $[11,12]$. Both $P$ and $N V$ belong to sixteen dimensional even and self-dual lattice $\Gamma_{16}$ or $\Gamma_{8} \times \Gamma_{8}$ depending on the gauge theory $\mathrm{SO}(32)$ or $E_{8} \times E_{8}$ [32]. Then we have unbroken gauge group and spectrum which are invariant under the orbifold projection.

The untwisted sector spectrum is obtained by

$$
\begin{aligned}
& \text { gauge bosons: } P \cdot V \equiv 0 \bmod 1, \\
& \text { untwisted matter: } P \cdot V \equiv \frac{\ell}{N} \quad \bmod 1, \quad \ell=1, \ldots\left\lfloor\frac{N}{2}\right\rfloor .
\end{aligned}
$$

With appropriate combinations from the right-movers we can make orbifold invariant state. In particular, the right mover has the phase $\phi_{1}= \pm \frac{1}{N}$ from the shift, thus the states with $P \cdot V \equiv \pm \frac{1}{N}$ modulo an integer can survive. This will be important property of six dimensional toroidal orbifold models.

\subsection{Perturbative modular invariance}

We require modular invariance of string partition function to divergence free, worldsheet reparametrization invariant theory. It is generated by so-called $S$ and $T$ generators of $\operatorname{SL}(2, \mathbb{Z})$ transformation of modular parameter $[11,12]$. Here we consider the invariance condition in the absence of heterotic 5-branes first.

Modular invariance under $S$ requires the existence of twisted strings, which are closed string up to orbifold action. Later we also see that twisted strings share many properties as open strings, whose ending hypersurface is interpreted as branes. We have $\lfloor N / 2\rfloor$ twisted sectors. In the $j$-th twisted sector the mass shell condition for the string left mover is

$$
\tilde{L}_{0}=0: \alpha^{\prime} m_{L}^{2}=\frac{(P+j V)^{2}}{2}+\tilde{\mathrm{N}}^{(j)}+E_{0}^{(j)}
$$

with the string tension $\alpha^{\prime}$ and the oscillator number $\tilde{\mathrm{N}}^{(j)}$, which is shifted, in the $j$ th twisted sector. This comes from the constraint of Virasoro generator $\tilde{L}_{0}=0$. It also includes the 
untwisted sector as $j=0$. The zero-point energy is given as

$$
E_{0}^{(j)}=-1+\frac{1}{2} \sum_{a=1}^{2} j \phi_{a}\left(1-j \phi_{a}\right) .
$$

Here, by $\phi_{a}^{(j)}$ we subtract an appropriate integer from $j \phi_{a}$ to let it lie in the interval $[0,1)$. Also with the right movers, satisfying

$$
L_{0}=0: \alpha^{\prime} m_{R}^{2}=\frac{(s+j \phi)^{2}}{2}+\mathrm{N}^{(j)}+E_{0}^{(j)}+\frac{1}{2},
$$

with a spacetime $\mathrm{SO}(8)$ weight vector $s$, we have localized fields at the fixed points. Here again $L_{0}$ is the Virasoro generator. With the right-mover, the whole state is subject to generalized GSO projection,

$$
e^{2 \pi i\left(\tilde{\mathrm{N}}-\mathrm{N}+(P+V) \cdot V-(s+\phi) \cdot \phi-\frac{1}{2}\left(V^{2}-\phi^{2}\right)\right)}
$$

by requiring this phase to be one [32-34].

Invariance under $T$ comes from the level matching condition [11, 12]

$$
\tilde{L}_{0}=L_{0}
$$

Consider the twisted sector. The condition (2.8) needs the following necessary condition

$$
\frac{(P+V)^{2}}{2}+\tilde{\mathrm{N}}-\frac{(s+\phi)^{2}}{2}-\mathrm{N}-\frac{1}{2}=0 \bmod 1 .
$$

In general there is no solution for the weight vector $P, s$ for a given $V, \phi$, because most of the terms proportional to $\frac{1}{N}$ cannot cancel the terms $\frac{1}{2}\left(V^{2}-\phi^{2}\right)$ proportional to $\frac{1}{2 N^{2}}$. It is necessary to require the former to be also proportional. This is so-called the modular invariance condition

$$
\frac{V^{2}}{2}-\frac{\phi^{2}}{2} \equiv 0 \quad \bmod \frac{1}{N}
$$

And the resulting vacua are sufficiently anomaly free $[42,43]$. The GSO phase is trivial when the massless condition is satisfied for modular invariant perturbative models.

\subsection{Classification of perturbative vacua}

In the orbifold embedding, the broken gauge group is parameterized by a shift vector. First consider $\mathrm{SO}(32)$ heterotic string. The shift vector of a type ${ }^{1}$

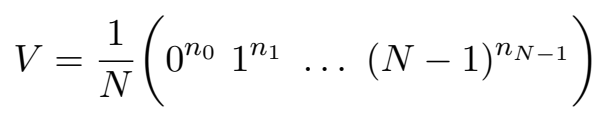

with

$$
\sum_{j=0}^{N-1} n_{j}=16
$$

\footnotetext{
${ }^{1}$ In our notation, the superscript in the shift vector denote the repeated entries.
} 
is called vectorial. This vector yields unbroken gauge group

$$
\begin{aligned}
& \mathbb{Z}_{2}: \mathrm{SO}\left(2 n_{0}\right) \times \mathrm{SO}\left(2 n_{1}\right) \\
& \mathbb{Z}_{3}: \mathrm{SO}\left(2 n_{0}\right) \times \mathrm{SU}\left(n_{1}+n_{2}\right) \times \mathrm{U}(1),
\end{aligned}
$$

according to (2.2). Note that the untwisted and twisted spectrum is the same if we use an equivalent shift vector under Weyl reflection accompanied by lattice translations. The gauge symmetry (2.14) can be partially explained by successive applications: if $n_{0}>0$, $\frac{1}{3}\left(0^{n_{0}} 1^{n_{1}} 2^{n_{2}}\right) \sim \frac{1}{3}\left(0^{n_{0}} 1^{n_{1}} 2^{n_{2}-2}-2-2\right) \sim \frac{1}{3}\left(0^{n_{0}} 1^{n_{1}+2} 2^{n_{2}-2}\right)$. In the $\mathbb{Z}_{2}$ case there is a gauge symmetry enhancement due to self-conjugate, giving (2.13).

There is another set of shift vectors, where $N V$ is a spinorial representation of $\Gamma_{16}$. Up to Weyl reflection and lattice translation, it can be generally expressed as

$$
V=\frac{1}{N}\left(\begin{array}{lll}
\frac{1}{2}^{m_{1}} & \frac{3}{2}^{m_{2}} & \ldots \frac{2 N-1}{2}^{m_{N}}
\end{array}\right)
$$

with

$$
\sum_{j=1}^{N} m_{j}=16 .
$$

In the special case of $\mathbb{Z}_{3}$ orbifold it can be always brought to vectorial shift by Weyl reflections accompanied by a lattice shift by $\left(\frac{1}{2}^{16}\right)$. There is no independent spinorial shift vector. In the $\mathbb{Z}_{2}$ orbifold however the spinorial shift vector cannot be reduced; it always gives the unbroken gauge group

$$
\mathbb{Z}_{2}: \mathrm{U}(16)
$$

For the $E_{8} \times E_{8}$ case, we form sixteen component vector by direct sum of two $\Gamma_{8}$ shift vectors, either vectorial or spinorial. In the $\mathbb{Z}_{2}$ and $\mathbb{Z}_{3}$ cases, the same argument as above tells us that the only vectorial shift vector combinations are sufficient

$$
V=\frac{1}{N}\left(0^{n_{0}} 1^{n_{1}} \ldots(N-1)^{n_{N-1}}\right)\left(0^{n_{0}^{\prime}} 1^{n_{1}^{\prime}} \ldots(N-1)^{n_{N-1}^{\prime}}\right)
$$

with

$$
\sum_{i=1}^{N-1} n_{i}=\sum_{i=1}^{N-1} n_{i}^{\prime}=8 .
$$

It is because up to Weyl reflections, we have three inequivalent groups in the $\mathbb{Z}_{2}$ case

$$
\begin{gathered}
\frac{1}{2}\left(0^{8}\right): E_{8}, \\
\frac{1}{2}\left(1^{2} 0^{6}\right) \sim \frac{1}{2}\left(\frac{1}{2}^{8}\right): E_{7} \times \mathrm{SU}(2), \\
\frac{1}{2}\left(1^{4} 0^{4}\right) \sim \frac{1}{2}\left(20^{7}\right): \mathrm{SO}(16),
\end{gathered}
$$


and five in the $\mathbb{Z}_{3}$ case

$$
\begin{gathered}
\frac{1}{3}\left(0^{8}\right): E_{8}, \\
\frac{1}{3}\left(1^{2} 0^{6}\right): E_{7} \times \mathrm{U}(1), \\
\frac{1}{3}\left(20^{7}\right): \mathrm{SO}(14) \times \mathrm{U}(1), \\
\frac{1}{3}\left(21^{2} 0^{5}\right): E_{6} \times \mathrm{SU}(3), \\
\frac{1}{3}\left(21^{4} 0^{3}\right): \mathrm{SU}(9) .
\end{gathered}
$$

Considering modular invariance condition the possibility reduces further. For $\mathrm{SO}(32)$ string with $N=3$, the condition becomes

$$
n_{1}+4 n_{2} \equiv 2 \bmod 6 .
$$

Without further symmetry breaking, e.g. by Wilson line, we have only five inequivalent modular invariant shift vectors for $\mathrm{SO}(32)$ [32] , as listed in table 1 for which we have $n=0$. For $E_{8} \times E_{8}$ string we have a similar condition,

$$
n_{1}+4 n_{2}+n_{1}^{\prime}+4 n_{2}^{\prime} \equiv 2 \bmod 6
$$

yielding also five combinations listed in table 2 with $n=0$. The shift vector for inequivalent, perturbative vacua are classified [19,35-37]. Thus the forms of the shift vectors (2.14) and (2.17) are sufficiently general in the $\mathbb{Z}_{3}$ case. We can do similar classification, yielding the spectrum in table 3 and table 4 .

\subsection{Instantons on $\mathbb{R}^{4} / \mathbb{Z}_{N}$ and $T^{4} / \mathbb{Z}_{N}$ orbifold}

The effect described by orbifold projection is captured by instanton background in the field theory limit $[17,21]$. The gauge group from heterotic string is broken by this instanton background.

Each fixed point of $T^{4} / \mathbb{Z}_{N}$ orbifold is locally described by $\mathbb{R}^{4} / \mathbb{Z}_{N}$ and is regarded as singular limit of the $A_{N-1}$ ALE space [22,30], which we discuss first. In the field theory limit, the following constant background cannot be gauged away due to orbifolding

$$
A=E \sum_{I=1}^{16} V_{I} H_{I} .
$$

Here $E$ is the exceptional divisor from the blown-up singularity, satisfying self-intersection relation $E \cdot E=-N$. The shift vector $V(2.11)$ has eigenvalues of the generator of the $\mathrm{SO}(32)$ group or $\mathrm{SO}(16) \times \mathrm{SO}(16)$ maximal torus subgroup of $E_{8} \times E_{8}$ carried by heterotic string [21]. They are generated by Cartan subalgebra elements $H_{I}$ 's.

In the orbifold limit, the unbroken group is enhanced to the group (2.14) of rank sixteen $[19,30]$, but the total instanton number should be preserved. Inside $V$ in (2.11) we 
have $n_{i}$ eigenvalue for each $i / N[13,21]^{2}$

$$
\begin{aligned}
k_{\mathbb{Z}_{N}} & =\frac{1}{8 \pi^{2}} \int_{A_{N-1} \text { ALE }} \operatorname{Tr} F^{2}=k_{\mathbb{Z}_{N}, \mathrm{U}}+k_{\mathbb{Z}_{N}, \mathrm{~T}}, \\
k_{\mathbb{Z}_{N}, \mathrm{U}} & =\frac{N}{2} \sum_{i=0}^{N-1} \frac{i}{N}\left(1-\frac{i}{N}\right) n_{i}=\frac{N}{2} \sum_{I=1}^{16} V_{I}\left(1-V_{I}\right), \\
k_{\mathbb{Z}_{N}, \mathrm{~T}} & \in \mathbb{Z} .
\end{aligned}
$$

Here $k_{\mathbb{Z}_{N}, \mathrm{U}}$ in $(2.25)$ is the usual second Chern class from the flat connection from the infinity. Roughly speaking, in $F^{2}$ we have $V_{I}$ contribution on one $F$ and $1-V_{I}$ contribution on the other $F$ from the structure of the generator $H_{I}[13,30]$. We may interpret that $k_{\mathbb{Z}_{N}, \mathrm{~T}}$ in (2.25) is the index of the localized fields at the tip of the ALE space. It can be an arbitrary number in the field theory, but the CFT of the global $T^{4} / \mathbb{Z}_{N}$ orbifold below completely determines it.

Now we wish to collect all the above local contributions to that on the compact $T^{4} / \mathbb{Z}_{N}$ orbifold. To this end, we should know the multiplicity of fixed points of possible orders of $N$. It is contained in the weights $d_{N}$, which are the number of $\mathbb{Z}_{N}$ invariant fixed points, and are determined by global structure of $T^{4} / \mathbb{Z}_{N}$ by relating Euler numbers of fixed points

$$
24=\chi\left(T^{4} / \mathbb{Z}_{N}\right)=d_{N} \chi\left(\mathbb{R}^{4} / \mathbb{Z}_{N}\right), N=2,3, \quad \chi\left(\mathbb{R}^{4} / \mathbb{Z}_{\ell}\right)=\frac{\ell^{2}-1}{\ell} .
$$

Therefore we have the total instanton numbers on $T^{4} / \mathbb{Z}_{N}$

$$
\begin{aligned}
& k_{\mathrm{U}}=d_{N} k_{\mathbb{Z}_{N}, \mathrm{U}}=d_{N} \frac{N}{2} \sum_{I=1}^{16} V_{I}^{(j)}\left(1-V_{I}^{(j)}\right), \\
& k_{\mathrm{T}}=d_{N} k_{\mathbb{Z}_{N}, \mathrm{~T}}=d_{N} k_{\mathbb{Z}_{N}, \mathrm{~T}},
\end{aligned}
$$

which are valid for $N=2,3$. Here again $V_{I}^{(j)}$ is $j V_{I}$ subtracted by an appropriate lattice vector to lie it in the interval $[0,1]$. In the presence of Wilson lines, the degeneracy from $d_{N}$ disappears and we have independent contribution from local shift vectors at each fixed point.

We see that $k_{\mathrm{U}}$ is a multiple of a fractional number, since the spectrum is (equally if there is no Wilson lines) distributed to a number of fixed points. For instance, in $T^{4} / \mathbb{Z}_{2}$ orbifold, the Euler number $\frac{3}{2}$ is equally distributed on the sixteen fixed points [22]. The bulk contribution is localized at the fixed points as well, in the orbifold limit.

For a $\mathbb{Z}_{N}$ shift vector of $\mathrm{SO}(32)$ in (2.11), we have contributions at each fixed point

$$
\begin{aligned}
& \mathbb{Z}_{2}: k_{\mathbb{Z}_{2}}=K_{2}+2 \cdot \frac{1}{2} \cdot \frac{1}{2} \cdot \frac{1}{2} \cdot n_{1}, \\
& \mathbb{Z}_{3}: k_{\mathbb{Z}_{3}}=K_{3}+3 \cdot \frac{1}{2} \cdot \frac{1}{3} \cdot \frac{2}{3} \cdot\left(n_{1}+n_{2}\right) .
\end{aligned}
$$

In the absence of Wilson lines, all the fixed points are equal, thus in the bulk we have

$$
\begin{aligned}
& \mathbb{Z}_{2}: k=16 K_{2}+4 n_{1}, \\
& \mathbb{Z}_{3}: k=9 K_{3}+3 n_{1}+3 n_{2} .
\end{aligned}
$$

\footnotetext{
${ }^{2}$ We use a different convention $w_{m}=2 n_{m}$ than that in the reference.
} 
Note that the instanton number is a gauge invariant quantity, as it should be. Either components $\frac{1}{3}$ or $\frac{2}{3}$ both describes $\mathrm{SU}(n)$.

For $E_{8} \times E_{8}$ string, the shift vector plays the same role: each component parameterizes the rotation in the Cartan subalgebra direction. Thus with the shift vector (2.17), we may use the same formula (2.26) for each $E_{8}$

$$
\begin{aligned}
& \mathbb{Z}_{2}: k_{\mathbb{Z}_{2}}=K_{2}+\frac{1}{4} n_{1}, \\
& \mathbb{Z}_{3}: k_{\mathbb{Z}_{3}}=K_{3}+\frac{1}{3}\left(n_{1}+n_{2}\right),
\end{aligned}
$$

where we count only the states charged under the first $E_{8}$. In the untwisted sector, usually a field is charged under one $E_{8}$. In the twisted sector, if a field is charged under the both, the representation becomes multiplicity. We have essentially the same counting for the second $E_{8}$. To sum up, we have

$$
\begin{aligned}
k & =k_{1}+k_{2}, \\
\mathbb{Z}_{2}: k_{1} & =16 K_{2}+4 n_{1}, k_{2}=16 K_{2}^{\prime}+4 n_{1}^{\prime} \\
\mathbb{Z}_{3}: k_{1} & =9 K_{3}+3 n_{1}+3 n_{2}, k_{2}=9 K_{3}^{\prime}+3 n_{1}^{\prime}+3 n_{2}^{\prime} .
\end{aligned}
$$

This establishes the relation between the modular invariance condition (2.10) and the Bianchi identity (1.1). We note that, although the condition (2.10) has similarity with the Bianchi identity (1.1), their contents are different. The shift vector and twist vector contain information of breaking of gauge symmetry and Lorentz symmetry, respectively, whereas the Bianchi identity contains the information about the spectrum. They are naturally related because the shift/twist vectors completely determine the spectrum.

\subsection{Chirality of localized fields}

In the smooth compactification, all the 24 small instantons reside in the bulk geometry of internal manifold. However in the toroidal orbifold, we have twisted fields localized at the fixed points and they carry instanton number $k_{\mathrm{T}}$ in (2.29) as well.

There exists no index theorem that automatically gives the spectrum in the twisted sector, yielding $k_{\mathrm{T}}$, because field theory cannot take into account the stringy nature of the twisted strings. We should calculate the twisted fields from CFT formulae (2.4) and (2.6).

Interestingly, for the $\mathrm{SO}(32)$ string, anomaly consideration of $\mathrm{SO}\left(2 n_{0}\right)$ gauge group is sufficient to fix the instanton number $k_{\mathrm{T}}$ for the twisted strings.

In the twisted sector, the contribution from twisted fields to the instanton number $k_{\mathrm{T}}$ depends on the type $\mathbf{R}$ of localized fields. Essentially it is determined by the twisted sector spectrum. Let $\mathbf{R}$ be representations of $\mathrm{SO}\left(2 n_{0}\right)$. Then the anomaly is related to the coefficient $x_{\mathbf{R}}$ in

$$
\operatorname{tr}_{\mathbf{R}} F^{4}=x_{\mathbf{R}} \operatorname{tr}_{\mathrm{v}} F^{4}+y_{\mathbf{R}}\left(\operatorname{tr}_{\mathrm{v}} F^{2}\right)^{2}
$$

where $\operatorname{tr}_{\mathrm{v}}$ is over the vectorial representation of $\mathrm{SO}\left(2 n_{0}\right)$. To sum up, in the perturbative model we have

$$
k_{\mathrm{T}}=\sum_{\mathbf{R} \text { for twisted fields }} x_{\mathbf{R}}
$$


This cancels six dimensional $\mathrm{SO}\left(2 n_{0}\right)$ gauge anomaly, with the chiral matter $\left(\mathbf{n}_{\mathbf{1}}+\mathbf{n}_{\mathbf{N}-\mathbf{1}}, \mathbf{2} \mathbf{n}_{\mathbf{0}}\right)$ under $\mathrm{SU}\left(n_{1}+n_{N-1}, 2 n_{0}\right)$ (or $\left(\mathbf{2} \mathbf{n}_{\mathbf{1}}, \mathbf{2} \mathbf{n}_{\mathbf{0}}\right)$ of $\mathrm{SO}\left(2 n_{1}, 2 n_{0}\right)$ for $\left.\mathbb{Z}_{2}\right)$

$$
\left(2 n_{0}-8\right)-n_{1}-n_{N-1}-k_{\mathrm{T}}=0,
$$

as long as the total instanton numbers should satisfy the condition (1.4)

$$
k=k_{\mathrm{U}}+k_{\mathrm{T}}=24 .
$$

It is because we can show the following using (2.12) and (2.32)

$$
\begin{aligned}
k_{\mathrm{T}} & =24-k_{\mathrm{U}} \\
& =-8+32-2 \sum_{i=1}^{N-1} n_{i}-n_{1}-n_{N-1} \\
& =-8+2 n_{0}-n_{1}-n_{N-1} .
\end{aligned}
$$

This proves the relation (2.36). The reason is that the total instanton number is related to the unbroken gauge group. Here the only unbroken part is $\mathrm{SO}\left(2 n_{0}\right)$.

In understanding the consistent vacua, the $\mathrm{SO}\left(2 n_{0}\right)$ anomaly plays an important role. In six dimension, the chirality of gaugino in the vector multiplet is always opposite to the fermions in the hypermultiplets, so that the matter contents are constrained by anomalies. Six dimensional gauge anomalies can be cancelled, up to Green-Schwarz mechanism, if the anomaly polynomial has vanishing tr $F^{4}$ term [21]. From the contributions of gaugino

$$
\begin{aligned}
\operatorname{Tr} F_{\mathrm{SO}(2 n)}^{4} & =(2 n-8) \operatorname{tr}_{\mathrm{v}} F_{\mathrm{SO}(2 n)}^{4}+6\left(\operatorname{tr} F_{\mathrm{SO}(2 n)}^{2}\right)^{2}, \\
\operatorname{Tr} F_{\mathrm{SU}(n)}^{4} & =2 n \operatorname{tr} F_{\mathrm{SU}(n)}^{4}+6\left(\operatorname{tr} F_{\mathrm{SU}(n)}^{2}\right)^{2},
\end{aligned}
$$

we see that we need $(2 n-8)$ vectors and $2 n$ fundamentals, respectively, to cancel $\mathrm{SO}(n)$ and $\mathrm{SU}(n)$ anomalies. Some other representations may contribute as follows. For the antisymmetric representations of $\mathrm{SU}(n)$ we have [14]

$$
\begin{aligned}
\operatorname{tr}_{\frac{\mathbf{n}(\mathbf{n}-\mathbf{1})}{\mathbf{2}}} F_{\mathrm{SU}(n)}^{4} & =(n-8) \operatorname{tr} F_{\mathrm{SU}(n)}^{4}+3\left(\operatorname{tr} F_{\mathrm{SU}(n)}^{2}\right)^{2}, \quad n \geq 4, \\
\operatorname{tr}_{\frac{\mathbf{n}(\mathbf{n}-\mathbf{1})(\mathbf{n}-\mathbf{2})}{\mathbf{6}}} F_{\mathrm{SU}(n)}^{4} & =\frac{1}{2}\left(n^{2}-17 n+54\right) \operatorname{tr} F_{\mathrm{SU}(n)}^{4}+(3 n-12)\left(\operatorname{tr} F_{\mathrm{SU}(n)}^{2}\right)^{2} .
\end{aligned}
$$

We have similar decompositions for the spinorial and antisymmetric of $\mathrm{SO}(2 n)$

$$
\begin{aligned}
\operatorname{tr}_{\mathbf{2}^{\mathbf{n}-1}} F_{\mathrm{SO}(2 n)}^{4} & =-2^{n-5} \operatorname{tr}_{\mathrm{v}} F_{\mathrm{SO}(2 n)}^{4}+3 \cdot 2^{n-5}\left(\operatorname{tr}_{\mathrm{v}} F^{2}\right)^{2}, \\
\operatorname{tr}_{\mathrm{v}} F_{\mathrm{SO}(2 n)}^{4} & =2 \operatorname{tr} F_{\mathrm{SU}(n)}^{4} .
\end{aligned}
$$

The absolute normalization is understood in the branching of vector $\mathrm{SO}(2 n)$ into fundamental of $\mathrm{SU}(n), \mathbf{2 n} \rightarrow \mathbf{n}+\overline{\mathbf{n}}$. The coefficients for $\operatorname{tr} F^{4}$ is zero for $\mathrm{SU}(n)$ with $n=2,3$ thus the corresponding groups are anomaly free.

It should be noted that the spinorial representation of $\mathrm{SO}\left(2 n_{0}\right)$ may have negative contribution to $k_{\mathrm{T}}$. Roughly 16 spinorial degrees of freedom contribute -1 , as seen from (2.42). We will see later that this makes some phase transitions difficult. 
The instanton number is different from other anomaly coefficients, for example of $\mathrm{SU}(n)$-type subgroups, or exceptional subgroups of $E_{8}$, or even $\mathrm{SO}\left(n_{1}\right)$ of the $\mathbb{Z}_{2}$ orbifold. However there is instanton number contribution for $k_{\mathrm{T}}$ from matter states.

$E_{8} \times E_{8}$ heterotic string vacua have a number of distinct features. There is no relation between anomaly and instanton numbers. Technically the zero entries of the shift vector do not yield $\mathrm{SO}\left(n_{0}\right)$ but different groups listed in (2.19) and (2.20). Here the instanton contribution comes from vectorial components of a representation, when we branch it into those of the maximal $\mathrm{SO}\left(n_{0}\right)$ group. For instance, $\mathbf{2 7}$ of $E_{6}$ contributes 1 and $\mathbf{5 6}$ of $E_{7}$ contributes 2 to the instanton number $k_{\mathrm{T}}$. Here the maximal $\mathrm{SO}\left(n_{0}\right)$ group is $\mathrm{SO}(10)$ with which we have branching $\mathbf{2 7} \rightarrow \mathbf{1 6}+\mathbf{1 0}+\mathbf{1}$. Thus formally we have the same relation as (2.34). We have no gauge symmetry enhancement from heterotic 5-branes, and any $E_{n}$ subgroups are anomaly free, thus the anomaly freeness is less restrictive.

\section{Non-perturbative vacua and their transitions}

Now we introduce heterotic 5-branes. As alluded in the introduction, there is phase transition between small instantons and 5-branes. Applyting this, it turns out that many seemingly unconnected vacua are actually connected. Since the effect of instantons is parameterized by the shift vector, we can trace the information on instanton exchange from the difference of the shift vectors of vacua. From this we can prove many relations among the zero point energy, the number of 5-branes and difference of the shift vectors.

\subsection{Small instantons into heterotic 5-branes}

The information about instanton embedding is encoded in the shift vector $V$. Some of instantons can be emitted into bulk to become heterotic 5-branes, recovering a larger unbroken group. The resulting unbroken group should be also described by another shift vector that we shall call $V_{1}$. Decomposing

$$
V=V_{1}+V_{2}
$$

the emitted instanton components are described by $V_{2}$, which describes also the recovered part.

Consider the twisted sector of a perturbative, modular invariant theory parameterized by $V$. Expanding the mass shell condition (2.4), we have

$$
\begin{aligned}
\frac{1}{2} \alpha^{\prime} m_{L}^{2} & =\frac{\left(P+V_{1}\right)^{2}}{2}+\tilde{\mathrm{N}}+P \cdot V_{2}+V_{1} \cdot V_{2}+\frac{1}{2} V_{2}^{2}+E_{0} \\
& =\frac{\left(P+V_{1}\right)^{2}}{2}+\tilde{\mathrm{N}}+E_{0}+\Delta E_{0} .
\end{aligned}
$$

We may regard this formula as the mass shell condition for the twisted sector for a daughter vacuum with $V_{1}$, with instantons emitted. Then the extra piece from the expanding $V$ can be regarded as modification in the zero-point energy

$$
\Delta E_{0} \equiv P \cdot V_{2}+V_{1} \cdot V_{2}+\frac{1}{2} V_{2}^{2} .
$$


Also the generalized GSO projector (2.7) can be expanded as

$$
e^{2 \pi i\left(\tilde{\mathrm{N}}-\mathrm{N}+\left(P+V_{1}\right) \cdot V_{1}-(s+\phi) \cdot \phi-\frac{1}{2}\left(V_{1}^{2}-\phi^{2}\right)+\Delta E_{0}\right)} .
$$

The extra phase is also simply expressed by $\Delta E_{0}$.

Note that the dependence of modified zero point energy (3.3) on $P$ means that the mass shell condition (3.2) is different for various representations. If all the twisted states satisfy $^{3}$

$$
P \cdot V_{2}=0,
$$

the zero point energy becomes constant

$$
\Delta E_{0} \equiv V_{1} \cdot V_{2}+\frac{1}{2} V_{2}^{2} .
$$

The spectrum can still be calculated using the same CFT in the presence of 5-branes, using the same formula as that of the perturbative case (3.2) [17]. The 5-branes can only affect the CFT on the fixed point only quantitatively. We may also expect that the number of 5 -branes should be related to $\Delta E_{0}$.

Note that the combination (3.6) is invariant under Weyl reflection. Indeed it is reflection that obviously preserves the inner product. Since the inclusion of heterotic 5-branes does not affect the internal geometry of orbifold, we have no change in the spacetime part, including that of the right mover. As a result, the part of instantons described by the shift vector $V_{2}$ 'condensates' in the CFT description. This justifies the shift of the zero point energy in ref. [17].

\subsection{Modified modular invariance condition}

The change of mass shell condition, now with the correction to the zero-point energy taking into account non-perturbative effects, affects the modular invariance condition. As in the perturbative case, we may consider level matching condition from the modified mass shell condition (3.2)

$$
\frac{(P+V)^{2}}{2}+\tilde{\mathrm{N}}+\Delta E_{0}-\frac{(s+\phi)^{2}}{2}-\mathrm{N}+\frac{1}{2}=0 \bmod 1 .
$$

Since each term is proportional to $1 / N$, we expect their cancellation gives an integer sum, expect the $V^{2}$ and $\phi^{2}$ terms. Thus we require the modified modular invariance condition

$$
\frac{V^{2}}{2}+\Delta E_{0}-\frac{\phi^{2}}{2} \equiv 0 \quad \bmod \frac{1}{N}
$$

\footnotetext{
${ }^{3}$ If we allow $P$-dependence on the zero point energy $E_{0}+\Delta E_{0}$, the only consistent way is to keep all the states from the mother theory with $V=V_{1}+V_{2}$ from (2.4). They are to recombine into the representation of the group $G_{1}$ of the daughter theory with $V_{1}$, as we see in the next section. However the resulting spectrum is (i) anomalous and/or (ii) cannot recombine into the complete representations of the gauge group $G_{1}$. For the states with $P \cdot V_{2} \not \equiv 0 \bmod 1$ we can make them projected out by (3.4) by removing $P \cdot V_{2}$ term in the phase. However we are not aware of the mechanism or condition to project out the state with $P \cdot V_{2} \equiv 0 \bmod 1$ which is not strictly $P \cdot V_{2}=0$. In general they form incomplete multiplets of $G_{1}$ thus are inconsistent.
} 
In the perturbative case we have $\Delta E_{0}=0$ giving the previous condition (2.10), but this fails in this case. Instead we require the form (3.8).

A shift vector $V$ that fails to satisfy perturbative modular invariance condition (2.10) can nevertheless describe a consistent non-perturbative vacuum [17]. In the $\mathrm{SO}(32)$ heterotic string theory, a failure of anomaly cancellation can be remedied by extra chiral fields coming from the heterotic 5-branes. In the $E_{8} \times E_{8}$ heterotic string, most vacua are anomaly free even without perturbative modular invariance.

We obtained the modular invariance condition (3.8) as a necessary condition. In the perturbative case that condition (2.10) also turns out to be sufficient condition. Unfortunately, this is not the case for the non-perturbative case. Even though we had shift vectors satisfying the condition we sometimes have anomalous vacua. We will come to a further selection rule and counterexample in section 3.7.

The modular invariance condition has preferred basis, so not every Weyl equivalent shift vector satisfies the same modular invariance condition. The modular invariance condition (2.21) is not symmetric under the exchange $n_{1}$ and $n_{2}$, although the unbroken group (2.14) is. It is rather a condition about the absolute size of the shift vector. The twisted sector mass shell condition (2.4) depends on it.

\subsection{Example}

Through this section, we consider an exemplar transition

- from the perturbative vacua of $G=\mathrm{U}(8) \times \mathrm{SO}(16)$ with the shift vector $V=\frac{1}{3}\left(1^{8} 0^{8}\right)$,

- to the non-perturbative vacua $G_{1}=\mathrm{U}(2) \times \mathrm{SO}(28)$ with the shift vectot $V_{1}=\frac{1}{3}\left(1^{2} 0^{14}\right)$.

They are found in table 1.

The mother theory has gauge group $G=\mathrm{U}(8) \times \mathrm{SO}(16)$, whose root vectors are

$$
\begin{aligned}
(64,1) & : P=\left(\underline{1-10^{6}} 0^{8}\right),(8 \text { oscillators }), \\
(\mathbf{1}, \mathbf{1 2 0}) & : P=\left(0^{8} \pm 1 \pm 10^{6}\right),(8 \text { oscillators }),
\end{aligned}
$$

where we allow permutations for underlined components. The matter states in the untwisted sector are

$$
\begin{aligned}
(\mathbf{8}, \mathbf{1 6}) & : P=\left(\underline{10^{7} \pm 10^{7}}\right), \\
(\mathbf{2 8}, \mathbf{1}) & : P=\left(\underline{-1-10^{6}} 0^{8}\right), \\
(\mathbf{1}, \mathbf{1}) & : G_{a \bar{a}}, a=1,2,
\end{aligned}
$$

which survive projection condition with appropriate right movers. The last ones are two $\mathbb{Z}_{3}$ invariant moduli fields from gravitons. The first twisted sector is the only twisted sector and has the mass shell condition (2.4),

$$
\frac{1}{2}(P+V)^{2}+\tilde{N}+E_{0}=0 .
$$


with the zero point energy

$$
E_{0}=-1+2 \cdot \frac{1}{2} \cdot \frac{1}{3} \cdot\left(1-\frac{1}{3}\right)=-\frac{7}{9} .
$$

Its solutions form

$$
\begin{aligned}
& 9(\mathbf{2 8}, \mathbf{1}): \tilde{N}=0, P=\left(\underline{-1-10^{6}} 0^{8}\right), \\
& 18(\mathbf{1}, \mathbf{1}): \tilde{N}=\frac{1}{3}, P=\left(0^{16}\right) .
\end{aligned}
$$

They survive the projection condition (2.7) with the massless right-movers. The multiplicity 9 is from the number of fixed points, while the extra factor 2 is the possible oscillator directions $\alpha_{-1 / 3}^{a}, a=1,2$.

The shift vector $V$ of $G$ is decomposed into $V_{1}$ of $G_{1}$ and an extra $V_{2}$ as

$$
V=V_{1}+V_{2}=\frac{1}{3}\left(1^{2} 0^{14}\right)+\frac{1}{3}\left(0^{2} 1^{6} 0^{8}\right) .
$$

Upon phase transition the small instanton components described by $V_{2}$ are going to become heterotic 5-branes. Therefore we are left with the remaining component $V_{1}$, describing a non-perturbative vacua with the gauge group $G_{1}$.

The unbroken gauge group is $\mathrm{U}(2) \times \mathrm{SO}(28)$, with untwisted matter

$$
\begin{aligned}
(\mathbf{2}, \mathbf{2 8}) & : P=\left(\underline{10} \pm 10^{13}\right), \\
(\mathbf{1}, \mathbf{1}) & : P=\left(\underline{-1-10^{6}} 0^{8}\right), \\
(\mathbf{1}, \mathbf{1}) & : G_{a \bar{a}}, a=1,2 .
\end{aligned}
$$

Instantons are embedded in the structure group $\mathbb{Z}_{3} \subset \mathrm{U}(1) \times \mathrm{SU}(2)$. It is described by the shift vector $V_{1}$, which is eventually related to the instanton number $k_{\mathrm{U}}=3 n_{1}=6$. In the twisted sector, on top of the usual zero-point energy $E_{0}$ in (3.9), there is modification as in (3.6),

$$
\Delta E_{0}=0+\frac{1}{2}\left(\frac{1}{3}\left(1^{6} 0^{10}\right)\right)^{2}=\frac{1}{3} .
$$

Plugging these into the mass shell condition (3.2), we find the spectrum ${ }^{4}$

$$
\begin{aligned}
9(\mathbf{1}, \mathbf{1}) & : \tilde{N}=0, P=\left(-1-10^{14}\right), \\
18(\mathbf{1}, \mathbf{1}): \tilde{N} & =\frac{1}{3}, P=\left(0^{16}\right) .
\end{aligned}
$$

\footnotetext{
${ }^{4}$ We see that these states are inherited from the mother theory (3.10). We have more inherited states

$$
\begin{array}{lll}
\tilde{N}=0, & P=\left(\underline{-10}-10^{3} 0^{10}\right), & P \cdot V_{2}=-\frac{1}{3}, \\
\tilde{N}=0, & P=\left(\begin{array}{lll}
00-1-100 & 0^{10}
\end{array}\right), & P \cdot V_{2}=-\frac{2}{3},
\end{array}
$$

and so on. They cannot form the complete representations of $\mathrm{SO}(28)$. We may project out these states if we remove $P \cdot V_{2}$ in the projector (3.4) because the phase $\left(P+V_{1}\right) \cdot V_{1}+P \cdot V_{2}$ is the same for all the inherited states from the mother theory with $V$. Only the state $P \cdot V_{2}=0$ in (3.13) survive. This is consistent with not having the massless state with $P \cdot V_{2} \neq 0$ in (3.2).
} 
Since there is no $\mathrm{SO}(28)$ vector or spinor, thus no instanton contribution comes from the twisted sector $k_{\mathrm{T}}=0$.

As a result, the 18 instantons described by $V_{2}$ are completely converted to as many $n=18$ heterotic 5 -branes by phase transition, so that the total number is conserved to be 24 as in (1.7). The biggest gauge group $\mathrm{Sp}(18)$ is generated if all the heterotic 5-branes become coincident. Besides the vector multiplets of both groups $\mathrm{SO}(28)$ and $\mathrm{Sp}(18)$, we have hypermultiplets in $(\mathbf{2}, \mathbf{1} ; \mathbf{3 6})$ and $\frac{1}{2}(\mathbf{1}, \mathbf{2 8} ; \mathbf{3 6})$. With the total 20 vectors, we can check that gauge anomalies of $\mathrm{SO}(28)$ cancel from (2.38).

In the above example, $V_{1} \cdot V_{2}=0$ happened, which can be relaxed in the following. There can be a transition between the perturbative $\mathrm{U}(5) \times \mathrm{SO}(22)$ model to nonperturbative $\mathrm{U}(4) \times \mathrm{SO}(24)$ model via

$$
\begin{aligned}
V & =\frac{1}{3}\left(\begin{array}{llll}
2 & 1^{4} & 0^{11}
\end{array}\right)=V_{1}+V_{2} \\
V_{1} & =\frac{1}{3}\left(\begin{array}{llllll}
1 & 1 & 1 & 1 & 0 & 0^{11}
\end{array}\right) \\
V_{2} & =\frac{1}{3}\left(\begin{array}{llllll}
1 & 0 & 0 & 0 & 1 & 0^{11}
\end{array}\right) .
\end{aligned}
$$

This gives the modification of zero-point energy $\Delta E_{0}=V_{1} \cdot V_{2}+\frac{1}{2} V_{2}^{2}=\frac{2}{9}$. This also lead us to anomaly-free vacuum. Alternatively, we can take another combination

$$
\begin{aligned}
V_{1}^{\prime} & =\frac{1}{3}\left(\begin{array}{lll}
0 & 1^{4} 0^{11}
\end{array}\right) \\
V_{2}^{\prime} & =\frac{1}{3}\left(\begin{array}{ll}
2 & \left.0^{15}\right) .
\end{array}\right.
\end{aligned}
$$

Remarkably the zero-point energy is corrected in the same amount $\Delta E_{0}=V_{1}^{\prime} \cdot V_{2}^{\prime}+\frac{1}{2} V_{2}^{\prime 2}=\frac{2}{9}$. The two models have the same spectrum. As long as $V_{1}$ and $V_{1}^{\prime}$ are related by Weyl transformation, we should always have the same $\Delta E_{0}$.

\subsection{Recombination of spectrum and anomaly flow}

Let us analyze the spectrum change, in the previous example, during the phase transition. First we see that the vector representations (in the hypermultiplets) in the untwisted sector of $G_{1}$ can be explained simply by branching and recombination from those of $G$. For this, it would be convenient to consider a common subgroup

$$
H \equiv \mathrm{U}(2) \times \mathrm{U}(6) \times \mathrm{SO}(16)
$$

which is a subgroup of both $G$ and $G_{1}$.

From the symmetry breaking $G \rightarrow H$, the spectrum branches as

$$
\begin{aligned}
& (\mathbf{8}, \mathbf{1 6}) \rightarrow(2,1,16)+(1,6,16) \\
& (\mathbf{2 8}, \mathbf{1}) \rightarrow(1,15,1)+(2,6,1)+(\mathbf{1}, \mathbf{1}, \mathbf{1}), \\
& (\mathbf{6 4}, \mathbf{1}) \rightarrow(4,1,1)+(1,36,1)+(2,6,1)+(2, \overline{6}, 1) .
\end{aligned}
$$

Note that only vector representations of $\mathrm{SO}(16) \subset G, H$ contribute to anomaly. We see that the only $\mathrm{SO}(16)$ vector representation is the multiplet $(\mathbf{8}, \mathbf{1 6})$ in the untwisted sector. There 
is no vector representation in the twisted sector. This multiplet and the untwisted state $(\mathbf{2 8}, \mathbf{1})$ are going to be recombined to the vector representation $\mathbf{2 8}$ of $\mathrm{SO}(28)$, responsible for anomaly. There are also nine $(\mathbf{2 8 , 1})$ states in the twisted sector and, after the branching and recombination, they are projected out except $(\mathbf{1}, \mathbf{1}, \mathbf{1})$ of $H$.

From the gauge symmetry enhancement $H \rightarrow G_{1}=\mathrm{U}(2) \times \mathrm{SO}(28)$, we have recombination

$$
\begin{aligned}
(2,28) \leftarrow & (2,6,1)+(2, \overline{6}, 1)+(2,1,16), \\
(\mathbf{1}, 378) \leftarrow & (\mathbf{1}, \mathbf{3 6}, \mathbf{1})+(\mathbf{1}, \mathbf{1}, \mathbf{1 2 0})+(\mathbf{1}, \mathbf{1 5}, \mathbf{1})+(\mathbf{1}, \overline{\mathbf{1 5}}, \mathbf{1}) \\
& +(\mathbf{1}, \mathbf{6}, \mathbf{1 6})+(\mathbf{1}, \overline{\mathbf{6}}, \mathbf{1 6}) .
\end{aligned}
$$

Among the eight vector representations $\mathbf{1 6}$ of $\mathrm{SO}(16)$ in (2.12), two are recombined into two 28 of $\mathrm{SO}(28)$ in (3.19). The other six 16's are un-higgsed and became the part of the adjoint of $\mathrm{SO}(28)$. Thus the number of vector representations is decreased by 6 , and we need $12=28-16$ more vector representation to compensate the anomaly of gauginos, thus 18 in total. They are provided by 18 emitted instantons becoming as many heterotic 5 -branes.

We can also keep track of anomaly flow during phase transition. We have derived the instanton number (2.32) from the field strength. Here, we can understand the multiplicative factors 3 in front of $n_{1}$ and $n_{2}$ in another way by looking at the spectrum change.

The emitted instantons are described by a part of the shift vector $V_{2}=\frac{1}{3}\left(\begin{array}{lll}0^{p} & 1^{q} & 0^{r}\end{array}\right)$ where $p+q+r=16$ (In the above case, we have $p=2, q=6, r=8$ ). Since $\mathrm{U}(p+q)$ became $\mathrm{U}(p)$, the number of vectors reduces by $q$ and thus the number of necessary heterotic 5branes for the anomaly cancellation of $\mathrm{SO}\left(2 n_{0}\right)$ increases by $2 q$. Thus the decreased small instanton is $3 q$, which should explain the coefficients 3 in front of $n_{1}$ and $n_{2}$ in eq. (2.32). Due to even root property of weight vector, $q$ should be multiple of 2 thus the number of extracted instantons should be always a multiple of 6 , as checked in tables 1 and 2 .

The same argument is possible for the transition from $\mathrm{SO}(2 n+4) \times \mathrm{SO}(2 m) \rightarrow \mathrm{SO}(2 n) \times$

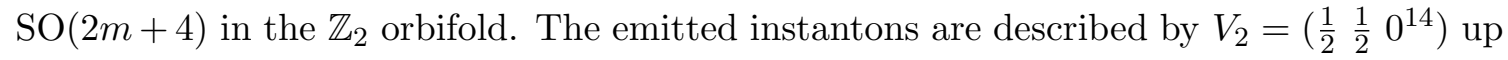
to permutation. The number of vector reduces by 4 but the necessary instantons increase by 4 , the coefficient of $n_{1}$ in eq. (2.31). Again due to the even nature of the lattice, the number of extracted instantons is always a multiple of 8 , as seen in tables 3 and 4 .

\subsection{Dual and inverse transitions}

The above phase transition always has a dual process. From the decomposition $V=V_{1}+V_{2}$, if we take a daughter model using the shift vector $V_{2}$, the emitted instanton data is contained in $V_{2}$, thus the zero-point energy is

$$
\Delta_{\text {dual }} E_{0}=V_{2} \cdot V_{1}+\frac{1}{2} V_{1} \cdot V_{1}
$$

For this dual transition, let us come to the example with (3.11). Exchanging the role of $V_{1}$ and $V_{2}$, we can view the transition as that of $\mathrm{U}(8) \times \mathrm{SO}(16) \rightarrow \mathrm{U}(6) \times \mathrm{SO}(20) \equiv G_{2}$ 
vacua. Six small instantons now described by $V_{1}$ may undergo transition into the same number of heterotic 5-branes. Accordingly, another recombination takes place

$$
\begin{aligned}
(6,20) & \leftarrow(\mathbf{1}, 6,16)+(\mathbf{2}, 6, \mathbf{1})+(\overline{2}, 6,1) \\
(\mathbf{1 5}, \mathbf{1}) & \leftarrow(\mathbf{1}, \mathbf{1 5}, \mathbf{1}) \\
(\mathbf{1}, \mathbf{1 9 0}) & \leftarrow(\mathbf{4}, \mathbf{1}, \mathbf{1})+(\mathbf{1}, \mathbf{1}, \mathbf{1 2 0})+(\mathbf{2}, \mathbf{1}, \mathbf{1 6})+(\overline{\mathbf{2}}, \mathbf{1}, \mathbf{1 6})+2(\mathbf{1}, \mathbf{1}, \mathbf{1}) .
\end{aligned}
$$

Again, we verify that the modified zero-point energy (3.21)

$$
\Delta E_{0}=V_{2} \cdot V_{1}+\frac{1}{2} V_{1}^{2}=\frac{1}{9}=\frac{6}{54} .
$$

This verifies also the transition of small instantons into $n=12$ heterotic 5 -branes. It should be noted that, however, for one transition $V_{1}$ yielding the same vacua, there can be many different dual vacua, as in the examples (3.14) and (3.15).

Reverse process, from a daughter theory with $V_{1}$ above to the mother theory with $V$, is also possible. If we express

$$
V_{1}=V-V_{2}
$$

then we can change the shift vectors of the mother and the daughter theories

$$
\begin{aligned}
\Delta_{\mathrm{rev}} E_{0} & =-V \cdot V_{2}+\frac{1}{2} V_{2}^{2} \\
& =-V_{1} \cdot V_{2}-\frac{1}{2} V_{2}^{2} \\
& =-\Delta E_{0} .
\end{aligned}
$$

Thus the process should be reversible.

\subsection{Phase transition of twisted states}

We also find phase transition of twisted states

$$
\Delta k_{\mathrm{T}} \neq 0
$$

In general, as we can see in tables $1-4$, the small instanton emission is accompanied by the change of the twisted states, resulting in the change (3.28). For instance, the transition from $V=\frac{1}{3}\left(21^{4} 0^{11}\right)$ to $V_{1}=\frac{1}{3}\left(1^{6} 0^{10}\right)$ changes not only the untwisted instanton number $k_{\mathrm{U}}$ but also the twisted one $k_{\mathrm{T}}$. The phase transition takes place preserving the sum

$$
24-k_{\mathrm{U}}-k_{\mathrm{T}}-n=0 .
$$

This is the result of the combining perturbative condition including twisted states (1.4) and non-perturbative condition with heterotic (1.7). This should be the result of combining the correspopnding Bianchi identities (1.3) and (1.6)

$$
\frac{1}{8 \pi^{2}} \operatorname{tr} \bar{R}^{2}-\sum_{a=1}^{n} \delta^{(4)}\left(x-x_{a}\right)-\frac{1}{8 \pi^{2}} \operatorname{Tr} \bar{F}_{\mathrm{U}}^{2}-\frac{1}{8 \pi^{2}} \operatorname{Tr} \bar{F}_{\mathrm{T}}^{2}=0 .
$$




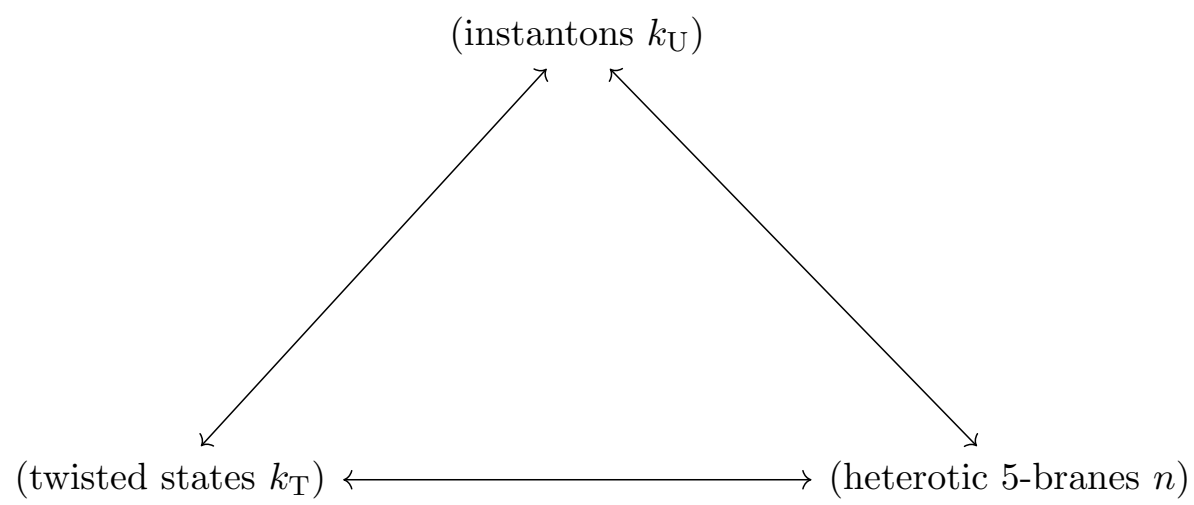

Figure 1. There are phase transitions among small instantons, heterotic 5-branes and twisted fields.

We have transition among small instantons, twisted states at orbifold fixed points, heterotic 5 -branes as in figure 1.

We may also have transition between untwisted and twisted instatons, not involving heterotic 5-branes. Since we have no control over the twisted sector fields via field theory, we cannot prove the direct transition. However, noting the untwisted sector and $k_{\mathrm{U}}$ are completely determined by the shift vector, we can indirectly use the chain of transitions. We may compare perturbative and non-perturbative vacua having the same shift vector. Such an example can be found in table 1 . The non-perturbative vacuum we have obtained from the transition in section 3.3 has

$$
V=\frac{1}{3}\left(1^{2} 0^{14}\right), \quad n=18 .
$$

However there is also a perturbative vacuum with

$$
V=\frac{1}{3}\left(1^{2} 0^{14}\right), \quad n=0
$$

The entire untwisted sector is the same in both cases, because we use the identical conditions for mass and projection. The gauge group is $\mathrm{U}(2) \times \mathrm{SO}(28)$ and the instanton number $k_{\mathrm{U}}=3 n_{1}=6$ are the same.

The modification to zero-point energy affects the twisted field spectrum. The $k_{\mathrm{T}}$ essentially counts the number vector multiplets $\mathbf{2 8}$ or equivalent in the twisted sector. We have $\mathrm{SU}(2)$ doublet in each of nine fixed points. Without Wilson line we do not distinguish among them. Therefore in the perturbative vacuum, we remove $2 \times 9=18$ vectors from the twisted sector. This should give rise to the same number of vectors from heterotic 5-branes in the non-perturbative model, satisfying (3.28).

The transition between fixed point states and 5-branes happens in general: if we have a transition between small instantons, changing $k_{\mathrm{U}}$, to heterotic 5-branes, there is also change in the twisted sector spectrum in general, changing $k_{\mathrm{T}}$. It is because the twisted spectrum is changed since the mass shell condition is changed as in (3.2). 
We can view the above exchange as dynamics of 5-branes. The dual description of small instantons is D5-branes on top of D9-branes. Twisted strings on the orbifold can also be regarded as open strings whose ends are interpreted as branes. These are localized $(5+1)$-dimensional hypersurface on which the twisted strings are localized [46, 47], in a similar sense that open strings that are localized on a NS5 or D5-brane. Moreover, we have seen that eq. (1.2) can be regarded as the magnetic equation for the $B$-field, in which the instantons and fixed points are all the magnetic sources.

\section{7 $\quad$ Selection rule}

We have seen phase transitions that are understood by exchange of 5-branes, in which the important constraint is the preserved number of 5 -branes as in the condition (1.7). In addition, this phase transition in orbifold theory takes place in a special form, as in eq. (3.2), namely that should be parameterized by the shift vector. Therefore the transition is further constrained by a selection rule that we shall see here.

Let us calculate the modification in the zero-point energy of $\mathbb{Z}_{3}$ vacua. They are described by shift vectors (2.11). The instanton number is given in (2.32) with $N=3$,

$$
k=9 K_{3}+3 n_{1}+3 n_{2}=24-n,
$$

where $K_{3} \equiv k_{\mathbb{Z}_{3}, \mathrm{~T}}$. There should be $n$ of heterotic 5-branes subject to global consistency condition (1.7). We have supposed that this vacuum is inherited from a perturbative modular invariant theory with

$$
k^{\mathrm{m}}=9 K_{3}^{\mathrm{m}}+3 n_{1}^{\mathrm{m}}+3 n_{2}^{\mathrm{m}}=24,
$$

where the superscripts indicate that the corresponding quantities are those of perturbative theory. The change of each contribution is described as follows,

$$
\Delta n_{i}=n_{i}-n_{i}^{\mathrm{m}}, \quad i=1,2, \quad \Delta K_{3}=K_{3}-K_{3}^{\mathrm{m}} .
$$

Note that, the change of instanton number is not only due to the change of the components $V_{1}$ but also the chirality of twisted sector spectrum. When small instantons get transition into heterotic 5-branes, both are related. Subtracting them we have

$$
9 \Delta K_{3}+3 \Delta n_{1}+3 \Delta n_{2}+n=0 .
$$

In the simplest case where the shift vector is simply decomposed $V=V_{1}+V_{2}$ and there is no overlapping elements, then the change of zero-point energy is obtained from (3.6)

$$
\Delta E_{0}=\frac{1}{2}\left(-\Delta n_{1}\left(\frac{1}{3}\right)^{2}-\Delta n_{2}\left(\frac{2}{3}\right)^{2}\right) \text {. }
$$


We may relate the number $n$ of heterotic 5-branes in (3.33) and the change $\Delta E_{0}$ of the zero point energy (3.34) as follows. In case $\Delta n_{1} \neq 0$ we may eliminate it to have

$$
\Delta E_{0}=\frac{n-9 \Delta K_{3}-9 \Delta n_{2}}{6 N^{2}},
$$

with $N=3$ here. This vacuum should be independent of transition, thus the modified zero point energy (3.35) should only be dependent on the number of heterotic 5-branes. It follows

$$
\Delta n_{2}+\Delta K_{3}=0 .
$$

We may have this same non-perturbative vacua from another perturbative vacuum with $\Delta n_{2} \neq 0$. However everything should be same if the transition is commutative. This means that we should always have

$$
n=-9 \Delta K_{3}-3 \Delta n_{2}-3 \Delta n_{1}=-3 \Delta n_{1}-12 \Delta n_{2}
$$

and

$$
\Delta E_{0}=\frac{n}{6 N^{2}},
$$

regardless of $\Delta n_{1}=0$ or not. Thus, the relation (3.36) provides a selection rule. This is nontrivial because, for a given shift vector, we cannot expect what kind of twisted fields can be obtained. In particular, if there is no change in the chirality in the twisted sector $\Delta K_{3}=0$, then the instanton described by entries with $n_{2}>0$ cannot be changed.

This selection rule (3.36) states that some transition is not possible. For instance, the phase transition from the perturbative vacuum with $V=\frac{1}{3}\left(1^{14} 0^{2}\right)$ to a non-perturbative with $V_{2}=\frac{1}{3}\left(1^{12} 0^{4}\right)$ is not possible. There is no change in $\Delta n_{2}$ thus we need $\Delta K_{3}=0$ from the rule (3.36). A stringy calculation using $V_{2}$ shows that we would have a $\mathrm{U}(12) \times \mathrm{SO}(8)$ vacuum with the twisted sector fields $9(\mathbf{6 6}, \mathbf{1})+18\left(\mathbf{1}, \mathbf{8}_{\mathbf{s}}\right)+18(\mathbf{1}, \mathbf{1})$ contributing $k_{\mathrm{T}}=$ $-9=9 \Delta K_{3} \neq 0$. Note that we have no way to predict the change and rearrangement of the twisted fields in the field theory limit so the selection rule is necessary condition. The resulting spectrum is anomalous, too. The would-be chain involving a non-perturbative vacuum with $V=\frac{1}{3}\left(1^{10} 0^{6}\right)$ is forbidden. This also shows that not every model satisfying the modified modular invariance condition (3.8) is not a sufficient condition in the non-perturbative vacua. The main reason is the anomaly contribution of spinorial representations. If we obtained the vacuum from the transition, the spinor representation cannot be well-branched under the transition (technically it can be seen from the mass formula in the twisted sector (2.4)). If we have only vector representations in the twisted sectors, it is likely that we have well-defined non-perturbative vacua.

This selection rule is based on some assumptions. First, assumed that all the fixed points are equivalent. This may be relaxed if we have Wilson lines. There can be a further complication due to the possibility of $V_{1} \cdot V_{2} \neq 0$. Also we assumed that there is no overlapping components between $V_{1}$ and $V_{2}$; otherwise the modification of the zero point energy $\Delta E_{0}$ has different dependence on $\Delta n_{i}$ 's. We will come back to this in the example below. Since this selection rule relates the number of instantons and the shift of zero point energy, it holds valid for $E_{8} \times E_{8}$ vacua. 


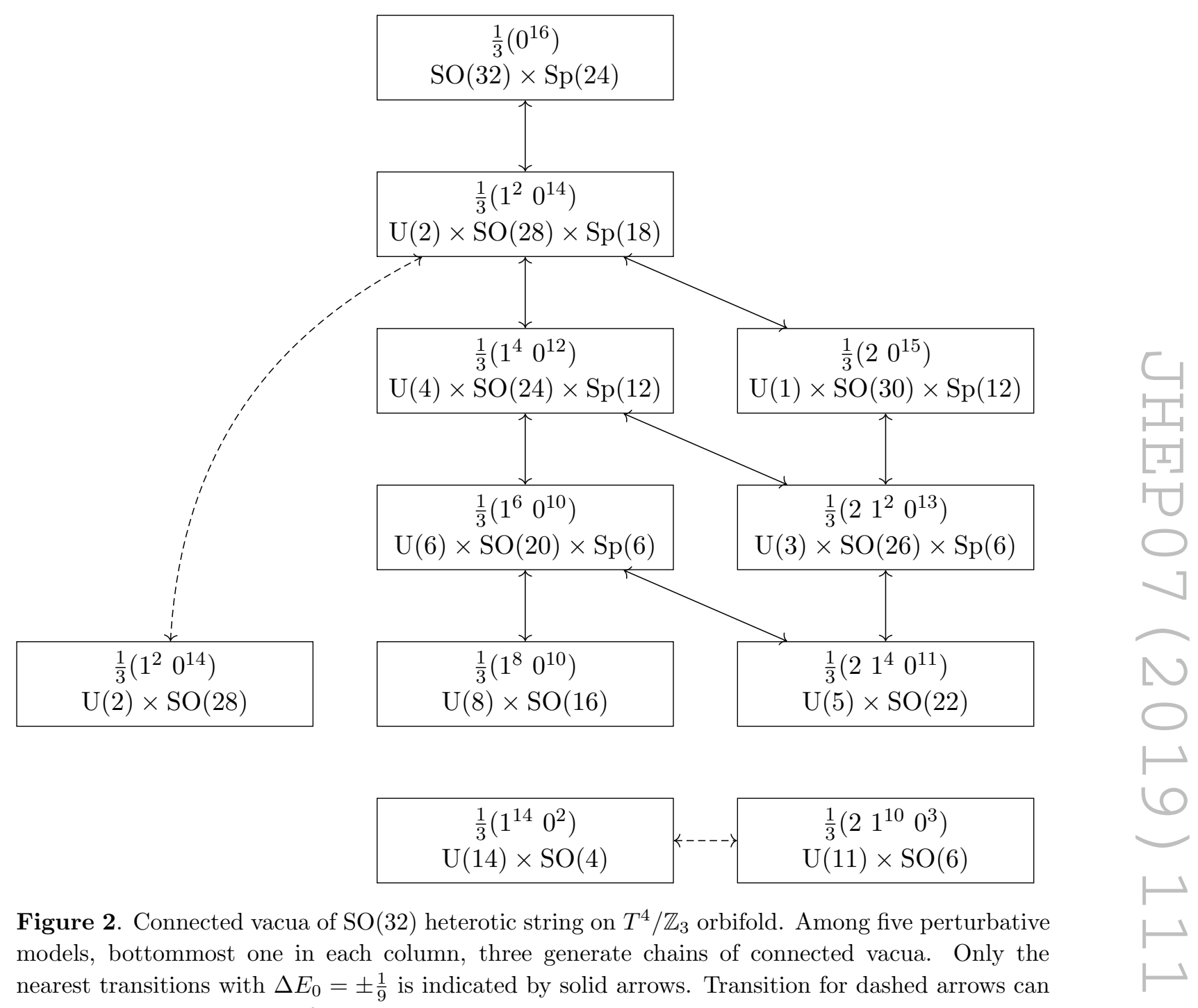
be proven using the chain of such transitions.

\section{Connected vacua}

The above discussion forces us to conclude that most of the vacua are connected. Chains of transitions of small instantons, heterotic 5-branes and twisted fields take one vacuum to another.

\subsection{Connected vacua of $\mathbb{Z}_{3}$ orbifold}

We consider global structure of moduli space. All the $\mathbb{Z}_{2}$ and $\mathbb{Z}_{3}$ vacua of both heterotic string theories are shown in tables $1-4$. The transition maps for the $T^{4} / \mathbb{Z}_{3}$ orbifold are depicted in figure 2 for $\mathrm{SO}(32)$ and in figure 3 for $E_{8} \times E_{8}$. Each heterotic string on this orbifold has five perturbative, modular invariant vacua. In $\mathrm{SO}(32)$ vacua two of them are disconnected and admit no transitions because the instanton number of the untwisted sector is too large. In these models, we have large spinorial representations contributing 


\begin{tabular}{|c|c|c|}
\hline Shift vector $V$ & Untwisted & $k_{\mathrm{U}}$ \\
\hline \multirow{2}{*}{ Group } & Twisted & $k_{\mathrm{T}}$ \\
\hline & heterotic 5 localized & $n$ \\
\hline$\frac{1}{3}\left(0^{16}\right)$ & $2(\mathbf{1} ; \mathbf{1})$ & 0 \\
\hline \multirow{2}{*}{$\mathrm{SO}(32) \times \mathrm{Sp}(24)$} & $18(\mathbf{1} ; \mathbf{1})$ & 0 \\
\hline & $\frac{1}{2}(32 ; 48)$ & 24 \\
\hline$\frac{1}{3}\left(20^{15}\right)$ & $(\mathbf{3 0} ; \mathbf{1})+2(\mathbf{1} ; \mathbf{1})$ & 3 \\
\hline \multirow{2}{*}{$\mathrm{U}(1) \times \mathrm{SO}(30) \times \mathrm{Sp}(12)$} & $9(\mathbf{3 0} ; \mathbf{1})+18(\mathbf{1} ; \mathbf{1})$ & 9 \\
\hline & $\frac{1}{2}(30 ; 24)$ & 12 \\
\hline$\frac{1}{3}\left(1^{2} 0^{14}\right)$ & $(\mathbf{2}, \mathbf{2 8} ; \mathbf{1})+3(\mathbf{1}, \mathbf{1} ; \mathbf{1})$ & 6 \\
\hline \multirow{2}{*}{$\mathrm{U}(2) \times \mathrm{SO}(28) \times \mathrm{Sp}(18)$} & $9(\mathbf{1}, \mathbf{1} ; \mathbf{1})+18(\mathbf{1}, \mathbf{1} ; \mathbf{1})$ & 0 \\
\hline & $\frac{1}{2}(\mathbf{1}, \mathbf{2 8} ; \mathbf{3 6})+(\mathbf{2}, \mathbf{1} ; \mathbf{3 6})$ & 18 \\
\hline$\frac{1}{3}\left(1^{2} 0^{14}\right)$ & $(\mathbf{2}, \mathbf{2 8})+3(\mathbf{1}, \mathbf{1})$ & 6 \\
\hline \multirow{2}{*}{$\mathrm{U}(2) \times \mathrm{SO}(28)$} & $9(\mathbf{2}, \mathbf{2 8})+18(\mathbf{1}, \mathbf{1})+45(\mathbf{1}, \mathbf{1})$ & 18 \\
\hline & & 0 \\
\hline$\frac{1}{3}\left(21^{2} 0^{13}\right)$ & $(\mathbf{3}, \mathbf{2 6} ; \mathbf{1})+(\mathbf{3}, \mathbf{1} ; \mathbf{1})+2(\mathbf{1}, \mathbf{1} ; \mathbf{1})$ & 9 \\
\hline \multirow{2}{*}{$\mathrm{U}(3) \times \mathrm{SO}(26) \times \mathrm{Sp}(6)$} & $9(\mathbf{1}, \mathbf{2 6} ; \mathbf{1})+9(\mathbf{1}, \mathbf{1} ; \mathbf{1})+18(\mathbf{3}, \mathbf{1} ; \mathbf{1})$ & 9 \\
\hline & $\frac{1}{2}(\mathbf{1}, \mathbf{2 6} ; \mathbf{1 2})+(\mathbf{3}, \mathbf{1} ; \mathbf{1 2})$ & 6 \\
\hline$\frac{1}{3}\left(1^{4} 0^{12}\right)$ & $(\mathbf{4}, \mathbf{2 4} ; \mathbf{1})+(\mathbf{6}, \mathbf{1} ; \mathbf{1})+2(\mathbf{1}, \mathbf{1} ; \mathbf{1})$ & 12 \\
\hline \multirow{2}{*}{$\mathrm{U}(4) \times \mathrm{SO}(24) \times \mathrm{Sp}(12)$} & $9(\mathbf{6}, \mathbf{1} ; \mathbf{1})+18(\mathbf{1}, \mathbf{1} ; \mathbf{1})$ & 0 \\
\hline & $\frac{1}{2}(\mathbf{1}, \mathbf{2 4} ; \mathbf{2 4})+(\mathbf{6}, \mathbf{1} ; \mathbf{2 4})$ & 12 \\
\hline$\frac{1}{3}\left(21^{4} 0^{11}\right)$ & $(\mathbf{5}, \mathbf{2 2})+(\mathbf{1 0}, \mathbf{1})+2(\mathbf{1}, \mathbf{1})$ & 15 \\
\hline \multirow{2}{*}{$\mathrm{U}(5) \times \mathrm{SO}(22)$} & $9(\mathbf{1}, \mathbf{2 2})+9(\mathbf{1 0}, \mathbf{1})+18(\mathbf{5}, \mathbf{1})$ & 9 \\
\hline & & 0 \\
\hline$\frac{1}{3}\left(1^{6} 0^{10}\right)$ & $(\mathbf{6}, \mathbf{2 0} ; \mathbf{1})+(\mathbf{1 5}, \mathbf{1} ; \mathbf{1})+2(\mathbf{1}, \mathbf{1} ; \mathbf{1})$ & 18 \\
\hline \multirow{2}{*}{$\mathrm{U}(6) \times \mathrm{SO}(20) \times \mathrm{Sp}(6)$} & $9(\mathbf{1 5}, \mathbf{1} ; \mathbf{1})+18(\mathbf{1}, \mathbf{1} ; \mathbf{1})$ & 0 \\
\hline & $\frac{1}{2}(\mathbf{1}, \mathbf{2 0} ; \mathbf{1 2})+(\mathbf{6}, \mathbf{1} ; \mathbf{1 2})$ & 6 \\
\hline$\frac{1}{3}\left(1^{8} 0^{8}\right)$ & $(\mathbf{8}, \mathbf{1 6})+(\mathbf{2 8}, \mathbf{1})+2(\mathbf{1}, \mathbf{1})$ & 24 \\
\hline \multirow{2}{*}{$\mathrm{U}(8) \times \mathrm{SO}(16)$} & $9(\mathbf{2 8}, \mathbf{1})+18(\mathbf{1}, \mathbf{1})$ & 0 \\
\hline & & 0 \\
\hline$\frac{1}{3}\left(21^{10} 0^{5}\right)$ & $(\mathbf{1 1}, \mathbf{1 0})+(\mathbf{5 5}, \mathbf{1})+2(\mathbf{1}, \mathbf{1})$ & 33 \\
\hline \multirow{2}{*}{$\mathrm{U}(11) \times \mathrm{SO}(10)$} & $9(\mathbf{1 1}, \mathbf{1})+9\left(\mathbf{1}, \mathbf{1 6}_{\mathrm{s}}\right)$ & -9 \\
\hline & & 0 \\
\hline$\frac{1}{3}\left(1^{14} 0^{2}\right)$ & $(\mathbf{1 4}, \mathbf{4})+(\mathbf{9 1}, \mathbf{1})+2(\mathbf{1}, \mathbf{1})$ & 42 \\
\hline \multirow[t]{2}{*}{$\mathrm{U}(14) \times \mathrm{SO}(4)$} & $9(\mathbf{1}, \mathbf{1})+9\left(\mathbf{1 4}, \mathbf{2}_{\mathrm{s}}\right)+18\left(\mathbf{1}, \mathbf{2}_{\mathrm{c}}\right)$ & -18 \\
\hline & & 0 \\
\hline
\end{tabular}

Table 1. Perturbative $(n=0)$ and non-perturbative $(n \neq 0)$ vacua of $\mathrm{SO}(32)$ string on $T^{4} / \mathbb{Z}_{3}$ orbifold. The parameters $k_{\mathrm{U}}, k_{\mathrm{T}}, n$ are respectively instanton numbers in the untwisted and twisted sectors, and the number of heterotic 5-branes, satisfying $k_{\mathrm{U}}+k_{\mathrm{T}}+n=24$. 


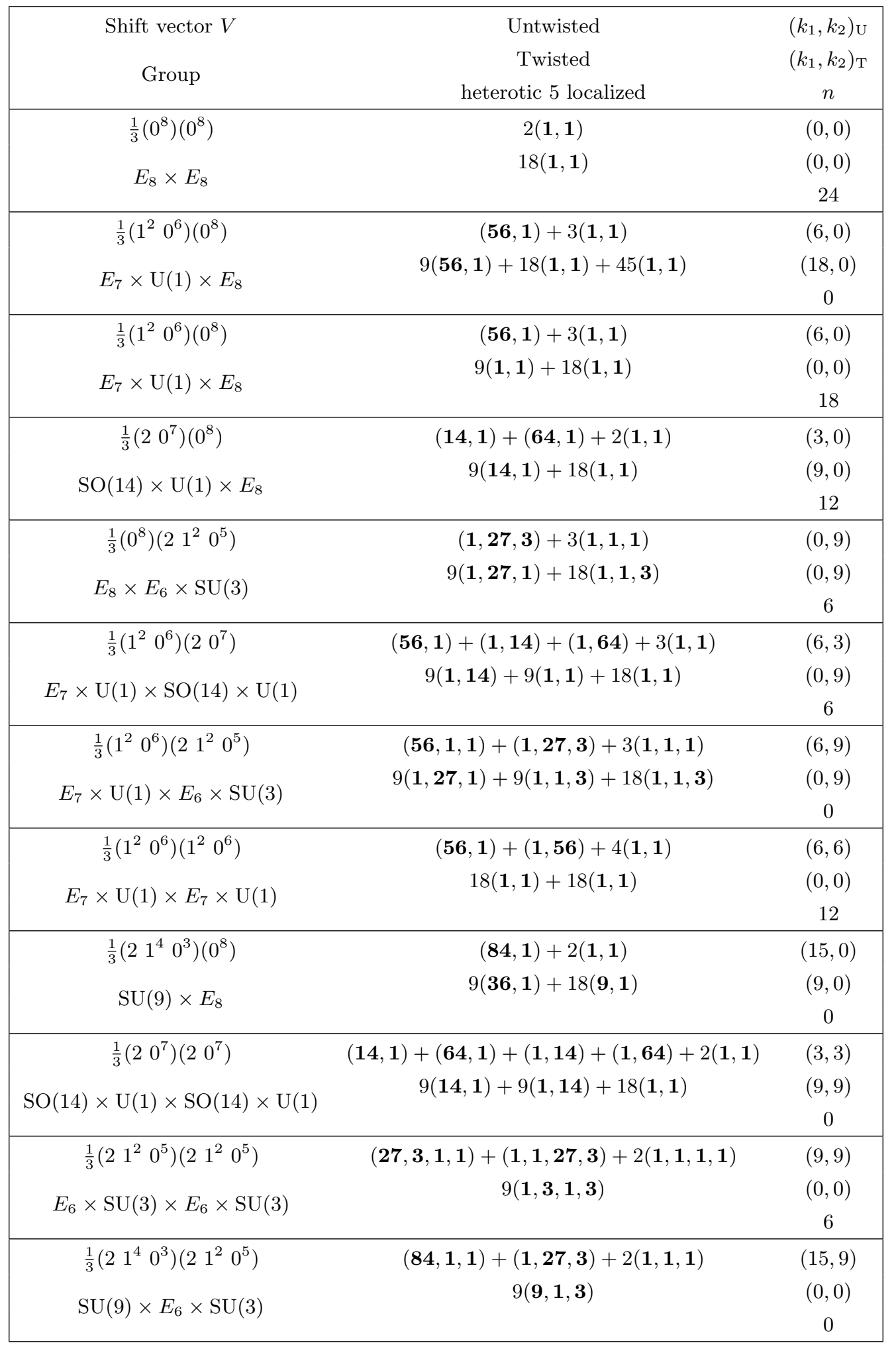

Table 2. Perturbative $(n=0)$ and non-perturbative $(n \neq 0)$ vacua of $E_{8} \times E_{8}$ heterotic string on $T^{4} / \mathbb{Z}_{3}$ orbifold. 


\begin{tabular}{|c|c|c|}
\hline Shift vector $V$ & Untwisted & $k_{\mathrm{U}}$ \\
\hline \multirow{2}{*}{ Group } & Twisted & $k_{\mathrm{T}}$ \\
\hline & heterotic 5 localized & $n$ \\
\hline$\frac{1}{2}\left(0^{16}\right)$ & $4(\mathbf{1} ; \mathbf{1})$ & 0 \\
\hline \multirow{2}{*}{$\mathrm{SO}(32) \times \mathrm{Sp}(24)$} & $18(\mathbf{1} ; \mathbf{1})$ & 0 \\
\hline & $\frac{1}{2}(32 ; 48)$ & 24 \\
\hline$\frac{1}{2}\left(1^{2} 0^{14}\right)$ & $(\mathbf{4}, \mathbf{2 8})+4(\mathbf{1}, \mathbf{1})$ & 8 \\
\hline \multirow{2}{*}{$\mathrm{SO}(4) \times \mathrm{SO}(28)$} & $8\left(\mathbf{2}_{\mathbf{c}}, \mathbf{2 8}\right)+32\left(\mathbf{2}_{\mathbf{s}}, \mathbf{1}\right)$ & 16 \\
\hline & & 0 \\
\hline$\frac{1}{2}\left(1^{2} 0^{14}\right)$ & $(\mathbf{4}, \mathbf{2 8} ; \mathbf{1})+4(\mathbf{1}, \mathbf{1} ; \mathbf{1})$ & 8 \\
\hline \multirow{2}{*}{$\mathrm{SO}(4) \times \mathrm{SO}(28) \times \mathrm{Sp}(16)$} & $8\left(\mathbf{2}_{\mathrm{s}}, \mathbf{1}\right)$ & 0 \\
\hline & $\frac{1}{2}(\mathbf{4}, \mathbf{1} ; \mathbf{3 2})+\frac{1}{2}(\mathbf{1}, \mathbf{2 8} ; \mathbf{3 2})$ & 16 \\
\hline$\frac{1}{2}\left(1^{4} 0^{12}\right)$ & $(\mathbf{8}, \mathbf{2 4} ; \mathbf{1})+4(\mathbf{1}, \mathbf{1} ; \mathbf{1})$ & 16 \\
\hline \multirow{2}{*}{$\mathrm{SO}(8) \times \mathrm{SO}(24) \times \mathrm{Sp}(8)$} & $8\left(\mathbf{8}_{\mathbf{s}}, \mathbf{1} ; \mathbf{1}\right)$ & 0 \\
\hline & $\frac{1}{2}(\mathbf{8}, \mathbf{1} ; \mathbf{1 6})+\frac{1}{2}(\mathbf{1}, \mathbf{2 4} ; \mathbf{1 6})$ & 8 \\
\hline$\frac{1}{2}\left(1^{6} 0^{10}\right)$ & $(\mathbf{1 2}, \mathbf{2 0})+4(\mathbf{1}, \mathbf{1})$ & 24 \\
\hline \multirow{2}{*}{$\mathrm{SO}(12) \times \mathrm{SO}(20)$} & $8\left(\mathbf{3 2} \mathbf{s}_{\mathrm{s}}, \mathbf{1}\right)$ & 0 \\
\hline & & 0 \\
\hline$\frac{1}{2}\left(\frac{1}{2}^{16}\right)$ & $2(\mathbf{1 2 0} ; \mathbf{1})+4(\mathbf{1} ; \mathbf{1})$ & 16 \\
\hline \multirow{2}{*}{$\mathrm{U}(16) \times \mathrm{Sp}(8)$} & $16(\mathbf{1} ; \mathbf{1})$ & 0 \\
\hline & $(16 ; 16)$ & 8 \\
\hline$\frac{1}{2}\left(\frac{1}{2}^{15}-\frac{3}{2}\right)$ & $2(\mathbf{1 2 0})+4(\mathbf{1})$ & 24 \\
\hline \multirow{2}{*}{$\mathrm{U}(16)$} & $16(\mathbf{1 6})$ & 0 \\
\hline & & 0 \\
\hline
\end{tabular}

Table 3. Perturbative $(n=0)$ and non-perturbative vacua of $\operatorname{SO}(32)$ heterotic string on $T^{4} / \mathbb{Z}_{2}$ orbifold. $k_{\mathrm{U}}, k_{\mathrm{T}}, n$ are small instanton number, the number of heterotic 5 -branes and the number of heterotic 5 -branes. These are all the inequivalent vacua satisfying the modular invariance condition up to Weyl reflections.

the instanton number $k_{\mathrm{T}}$ in the twisted sector. After the transition, in the presence of heterotic 5-brane, we have large positive shift of the zero point energy, hence it is difficult to have still the spinorial representation, making transition impossible.

Starting from one vacuum, we can go to another by emitting small instantons into heterotic 5-branes. We can travel every solid arrow by transition parameterized by auxiliary shift vector

$$
V_{2}=\frac{1}{3}\left(0^{a} \pm 1 \pm 10^{b}\right), \quad a+b=14 .
$$

We may go directly to any of them by ejecting a multiple of six instantons at the same time. In particular in $\mathrm{SO}(24) \times \mathrm{U}(2)$ model there is a transition between twisted fields and heterotic 5-branes, whose direction is denoted by dashed arrow. In all of the above transitions, we have the relation between the shift vector and emitted instanton number

$$
\Delta E_{0}=V_{1} \cdot V_{2}+\frac{1}{2} V_{2}^{2}=\frac{\Delta n}{6 N^{2}}
$$




\begin{tabular}{|c|c|c|}
\hline $\begin{array}{l}\text { Shift vector } V \\
\text { Group }\end{array}$ & $\begin{array}{c}\text { Untwisted } \\
\text { Twisted } \\
\text { heterotic } 5 \text { localized }\end{array}$ & $\begin{array}{c}\left(k_{1}, k_{2}\right)_{\mathrm{U}} \\
\left(k_{1}, k_{2}\right)_{\mathrm{T}} \\
n\end{array}$ \\
\hline$\frac{1}{2}\left(0^{8}\right)\left(0^{8}\right)$ & $4(\mathbf{1} ; \mathbf{1})$ & $(0,0)$ \\
\hline$E_{8} \times E_{8}$ & $8(\mathbf{1} ; \mathbf{1})$ & $\begin{array}{c}(0,0) \\
24\end{array}$ \\
\hline$\frac{1}{2}\left(1^{2} 0^{6}\right)\left(0^{8}\right)$ & $(\mathbf{5 6}, \mathbf{2})+4(\mathbf{1}, \mathbf{1})$ & $(8,0)$ \\
\hline$E_{7} \times \mathrm{SU}(2) \times E_{8}$ & $8(\mathbf{5 6}, \mathbf{1})+32(\mathbf{1}, \mathbf{2})$ & $\begin{array}{c}(16,0) \\
0\end{array}$ \\
\hline$\frac{1}{2}\left(1^{2} 0^{6}\right)\left(0^{8}\right)$ & $(\mathbf{5 6}, \mathbf{2})+4(\mathbf{1}, \mathbf{1})$ & $(8,0)$ \\
\hline$E_{7} \times \mathrm{SU}(2) \times E_{8}$ & $8(\mathbf{5 6}, \mathbf{1})+16(\mathbf{1}, \mathbf{1})$ & $\begin{array}{c}(0,0) \\
16\end{array}$ \\
\hline $\begin{aligned} & \frac{1}{2}\left(1^{2} 0^{6}\right)\left(1^{2} 0^{6}\right) \\
& E_{7} \times \mathrm{SU}(2) \times E_{7} \times \mathrm{SU}(2)\end{aligned}$ & $\begin{array}{c}(\mathbf{5 6}, \mathbf{2}, \mathbf{1}, \mathbf{1})+(\mathbf{1}, \mathbf{1}, \mathbf{5 6}, \mathbf{2})+4(\mathbf{1}, \mathbf{1}, \mathbf{1}, \mathbf{1}) \\
8(\mathbf{1}, \mathbf{2}, \mathbf{1}, \mathbf{2})\end{array}$ & $\begin{array}{c}(8,8) \\
(0,0) \\
8\end{array}$ \\
\hline $\begin{array}{l}\frac{1}{2}\left(0^{8}\right)\left(1^{4} 0^{4}\right) \\
E_{8} \times \operatorname{SO}(16)\end{array}$ & $\begin{array}{c}(\mathbf{1}, \mathbf{1 2 8})+4(\mathbf{1}, \mathbf{1}) \\
8(\mathbf{1}, \mathbf{1 6})\end{array}$ & $\begin{array}{c}(0,16) \\
(0,0) \\
8\end{array}$ \\
\hline $\begin{array}{c}\quad \frac{1}{2}\left(1^{2} 0^{6}\right)\left(1^{4} 0^{4}\right) \\
E_{7} \times \mathrm{SU}(2) \times \mathrm{SO}(16)\end{array}$ & $\begin{array}{c}(\mathbf{5 6}, \mathbf{2}, \mathbf{1})+(\mathbf{1}, \mathbf{1}, \mathbf{1 2 8})+4(\mathbf{1}, \mathbf{1}, \mathbf{1}) \\
8(\mathbf{1}, \mathbf{2}, \mathbf{1 6})+16(\mathbf{1}, \mathbf{1}, \mathbf{1})\end{array}$ & $\begin{array}{c}(8,16) \\
(0,0) \\
0 \\
\end{array}$ \\
\hline
\end{tabular}

Table 4. Perturbative $(n=0)$ and non-perturbative vacua of $E_{8} \times E_{8}$ heterotic string on $T^{4} / \mathbb{Z}_{2}$ orbifold.

generalizing (3.38). (For the transition from a mother theory with $V=V_{1}+V_{2}$ to a daughter theory with $V_{1}$ we have always $V_{1} \cdot V_{2}=0$.) The reverse transition is possible so the transition is two way. Note that there can be a direct transition between two vacua even if they are not directly connected by an arrow. For instance, we have seen that we can go directly from $\frac{1}{3}\left(1^{8} 0^{8}\right)$ vacuum to $\frac{1}{3}\left(1^{2} 0^{14}\right)$ vacuum.

This argument may hold for two vacua with the same instanton numbers. Consider for example $V=\frac{1}{3}\left(1^{4} 0^{12}\right)$ and $V_{1}=\frac{1}{3}\left(20^{15}\right)$ are horizontally connected by chains of transitions. We can describe their direct connection using $V_{2}=\frac{1}{3}\left(\begin{array}{llllllll}-1 & 1 & 1 & 1 & 0^{12}\end{array}\right)$, giving $\Delta E_{0}=V_{1} \cdot V_{2}+\frac{1}{2} V_{2}^{2}=0$. If they are directly connected by virtual instanton exchanges, we may expect that the bottommost two vacua of $\mathrm{SO}(32)$, with $\frac{1}{3}\left(1^{14} 0^{2}\right)$ and $\frac{1}{3}\left(21^{10} 0^{3}\right)$ could be connected by transition as well.

When all the 24 small instantons are emitted and become coincident we have the maximal gauge group $\mathrm{Sp}(24)$. The biggest 'bifundamental' representation is $(32,48)$ in the half multiplet. Note that in this case, the duality between $E_{8} \times E_{8}$ and $\mathrm{SO}(32)$ works, because all the spectra have one-to-one correspondence. The brainy picture in type I side has been well-studied [40, 41, 44].

For the $E_{8} \times E_{8}$ models, we have two groups of connected vacua. In the $E_{8}$ case, the shift vector $V=\frac{1}{3}\left(20^{7}\right)\left(0^{8}\right)$ is equivalent to $V^{\prime}=\frac{1}{3}\left(1^{4} 0^{4}\right)\left(0^{8}\right)$ by Weyl reflection. Starting from either vector we can obtain the descendent model with $V_{1}=\frac{1}{3}\left(1^{2} 0^{6}\right)\left(0^{8}\right)$ by 

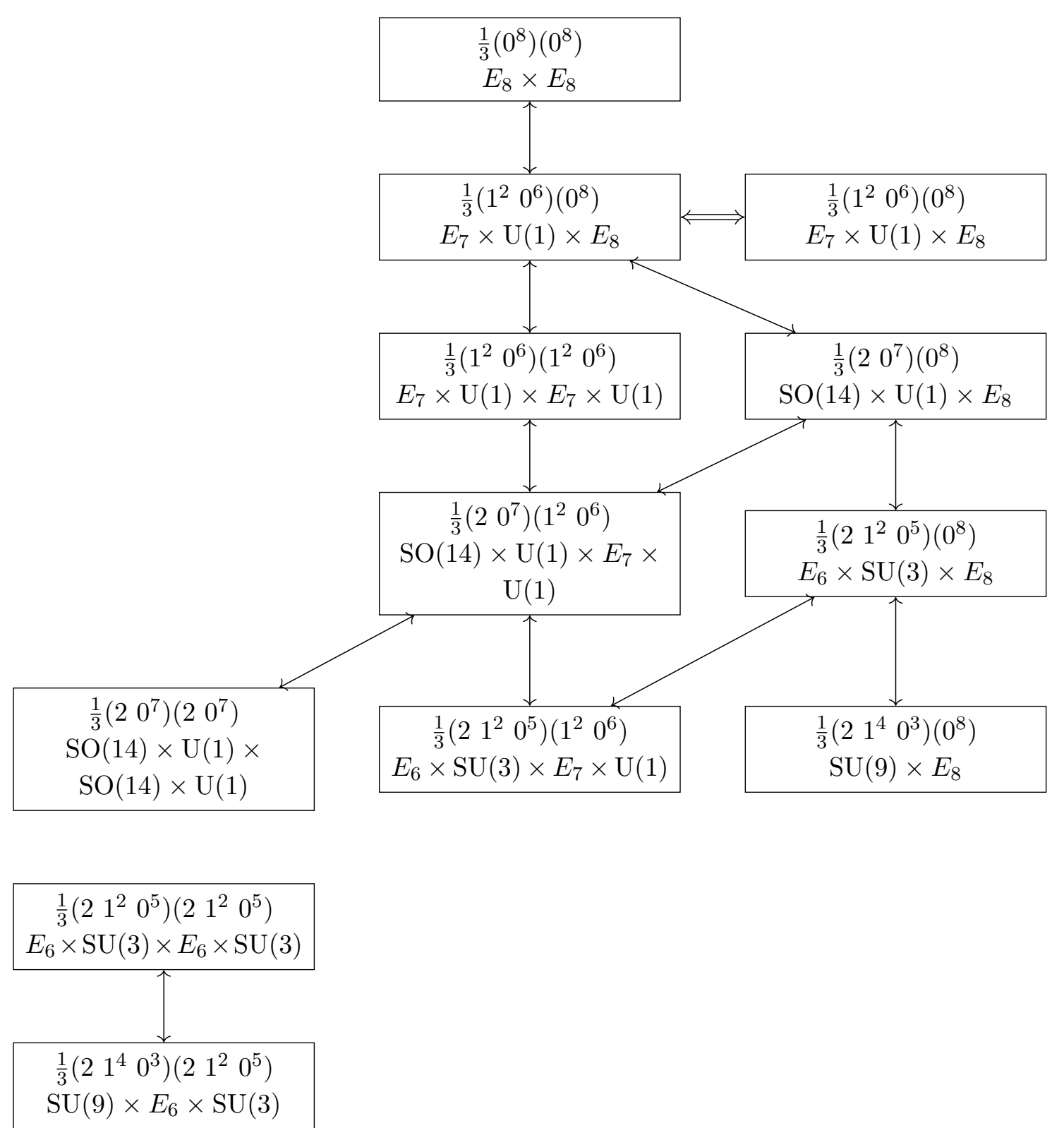

Figure 3. Connected vacua of $E_{8} \times E_{8}$ heterotic string on $T^{4} / \mathbb{Z}_{3}$ orbifold. Among five perturbative models, bottommost one in each column, three generate chains of connected vacua.

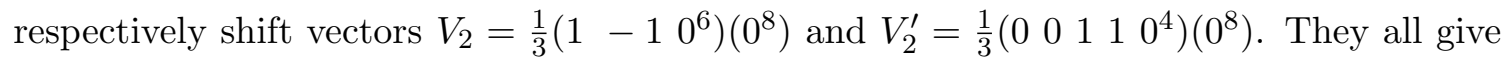
the same zero point energy correction $\Delta E_{0}=V_{1} \cdot V_{2}+\frac{1}{2} V_{2}^{2}=V_{1}^{\prime} \cdot V_{2}^{\prime}+\frac{1}{2} V_{2}^{\prime 2}$.

There are transitions between perturbative vacua, in which small instantons are exchanged to twisted fields. We have shown this using the chain of dualities.

Note that there is duality between two heterotic string theories. At the tip of the chains of each string theory, we have the same field contents. So the two heterotic string theories are dually related. 


\begin{tabular}{|ccc|}
\hline Shift vector $V$ & Untwisted & $\left(k_{1}, k_{2}\right)_{\mathrm{U}}$ \\
Group & Twisted & $\left(k_{1}, k_{2}\right)_{\mathrm{T}}$ \\
& heterotic 5 localized & $n$ \\
\hline$\frac{1}{3}\left(21^{2} 0^{5}\right)\left(20^{7}\right)$ & $(\mathbf{2 7}, \mathbf{3}, \mathbf{1}, \mathbf{1})+(\mathbf{1}, \mathbf{1}, \mathbf{1 4}, \mathbf{1})+(\mathbf{1}, \mathbf{1}, \mathbf{6 4}, \mathbf{1})+2(\mathbf{1}, \mathbf{1}, \mathbf{1}, \mathbf{1})$ & $(9,3)$ \\
$E_{6} \times \mathrm{SU}(3) \times \mathrm{SO}(14) \times \mathrm{U}(1)$ & $9(\mathbf{1}, \mathbf{3}, \mathbf{1}, \mathbf{1})$ & $(0,0)$ \\
\hline$\frac{1}{3}\left(21^{4} 0^{3}\right)\left(20^{7}\right)$ & $(\mathbf{8 4}, \mathbf{1})+(\mathbf{1}, \mathbf{1 4})+(\mathbf{1}, \mathbf{6 4})+2(\mathbf{1}, \mathbf{1})$ & $(15,3)$ \\
$\mathrm{SU}(9) \times \mathrm{SO}(14) \times \mathrm{U}(1)$ & $9(\mathbf{9}, \mathbf{1})$ & $(0,0)$ \\
\hline$\frac{1}{3}\left(21^{2} 0^{5}\right)\left(21^{2} 0^{5}\right)$ & & 6 \\
$E_{6} \times \mathrm{SU}(3) \times E_{6} \times \mathrm{SU}(3)$ & $(\mathbf{2 7}, \mathbf{3}, \mathbf{1}, \mathbf{1})+(\mathbf{1}, \mathbf{1}, \mathbf{2 7}, \mathbf{3})+2(\mathbf{1}, \mathbf{1}, \mathbf{1}, \mathbf{1})$ & $(9,9)$ \\
& $9(\mathbf{1}, \mathbf{3}, \mathbf{1}, \mathbf{3})$ & $(0,0)$ \\
\end{tabular}

Table 5. (Possibly) inconsistent vacua of $E_{8} \times E_{8}$ heterotic string on $T^{4} / \mathbb{Z}_{3}$ orbifold, but passing the selection rule.

\subsection{Communication between two $E_{8}$ 's}

In many cases, the selection rule (3.36) applies well to supplement the modular invariance condition. For example, transition from $V=\frac{1}{3}\left(21^{4} 0^{3}\right)\left(21^{2} 0^{5}\right)$ to $V_{1}=\frac{1}{3}\left(21^{4} 0^{3}\right)\left(1^{2} 0^{6}\right)$ is not possible because while $\Delta n_{2}=1$ we have $\Delta K_{3}=0$. However there are inconsistent $E_{8} \times E_{8}$ vacua even they pass both conditions; the resulting spectra are anomalous. They are listed in table 5 .

For example, the model $V_{1}^{\prime}=\frac{1}{3}\left(21^{4} 0^{3}\right)\left(20^{7}\right)$ is anomalous although the transition from the above vacuum with $V$ seems possible according to the selection rule from $\Delta K_{3}=$ $\Delta n_{2}=0$. It would be anomaly free if some twisted fields are branched from those in the vacuum with $V$. The desired representation does not arise in the twisted sector because the modification of the zero point energy is too large for this representation to satisfy the mass shell condition.

Another example is a seemingly consistent vacuum with

$$
\frac{1}{3}\left(21^{2} 0^{5}\right)\left(21^{2} 0^{5}\right) \text { giving } E_{6} \times \mathrm{SU}(3) \times E_{6} \times \mathrm{SU}(3)
$$

It is not anomalous because the unbroken gauge groups are anomaly free by default. Note that this vacuum is an intermediate one along the chain of transition from $V=$ $\frac{1}{3}\left(21^{4} 0^{3}\right)\left(21^{2} 0^{5}\right)$ to the above inconsistent vacua, so this may be inconsistent as a string model although it is field theoretically anomaly free.

One interesting observation is that, except this one with (4.2), all the non-perturbative vacua in the presence of heterotic 5-branes contain no charged field under two groups originating from different $E_{8}$ 's. The above anomalous model, which satisfy the modified modular invariance condition and pass the selection rules, requires charged field under two groups coming from different $E_{8}$ 's. This provides a suggestive explanation on the communication problem between two $E_{8}$ 's, and deserves further study [45]. By emitting small instantons in one $E_{8}$ and absorbing them in the other $E_{8}$, chiralities can be exchanged. Thus these two $E_{8}$ can be exchanged. 
In the strong coupling limit we open up a new interval that separates these two $E_{8}$ 's. The 5-brane is interpreted as M5-brane if we regard this theory as M-theory compactified on an interval and after phase transition this can be emitted in the bulk as explained in the introduction. Therefore M5-branes can be messengers for exchanging chirality between the two $E_{8}$ 's. The technical reason making sense of this argument is that if we have nonzero number $n$ of heterotic 5-branes, this introduces modification of the zero point energy, which makes hard to satisfy nontrivial mass shell condition. In this situation, typically it is very difficult to have a charged state under two groups inherited from different $E_{8}$ 's, which partly justify the above observation. This would let us to rule out the vacuum (4.2). It would be also interesting for further study if this transition should always be involved in communicating two $E_{8}$ 's in the strong coupling limit of $E_{8} \times E_{8}$ heterotic string.

\section{Discussion}

We have studied vacua of heterotic string compactified on $T^{4} / \mathbb{Z}_{N}$ orbifold, with $N=2,3$, in the presence of heterotic 5-branes. A new understanding comes from phase transition between small instantons and 5-branes. It does not only explain the spectrum, but also suggests that most of vacua of toroidal orbifolds are connected. This hints at the evolution of stringy vacua in the landscape.

In the presence of heterotic 5-branes, although the theory is non-perturbative, we have still exactly solvable CFT in the worldsheet. This is because the 5-branes can be obtained by instanton transition from a perturbative vacuum, which is indeed described by a solvable CFT. The effect of heterotic 5-brane modifies the zero-point energy [17]. Rather than previous bottom-up approach, we can derive the modified zero-point energy using phase transition between small instantons and 5-branes. With this, we derive modified modular invariance condition. This gives us deeper understanding on the relation between the modular invariance and Bianchi identity.

The most striking feature is the connection between two perturbative vacua, in which some small instantons are exchanged to twisted sector fields. Comparison of vacua also suggests that orbifold fixed points also behave like branes. Therefore we may have transition among small instantons, heterotic 5-branes and twisted states. Although we have used indirect chains of transitions, it would be interesting to study any direct transition mechanism. This is another feature that twisted fields behave like open strings whose boundary conditions are described by heterotic 5-branes. We might have a fuller brainy description for the orbifold fixed points.

We note that some vacua that satisfy the modified modular invariance condition in the presence of heterotic 5-branes give anomalous spectra. Contrary to the perturbative case, the modular invariance condition is not the sufficient condition for the consistency. We still do not know fully sufficient condition for anomaly cancellation in the presence of 5-branes. This is due to lack of the full understanding on the twisted fields; we do not know how they originate, e.g. from branching of a larger representation but at best we obtain them from CFT equation. In the smooth case, ref. [10] calculated the modular invariance condition 
for $E_{8} \times E_{8}$ heterotic orbifolds including the 5-brane effects, using the topological vertex and the total instanton number condition is the sufficient condition.

In this paper, we have focused on the transition mechanism and treated every fixed point is equal. If we have Wilson lines, we may relax the condition. In this way we hope to find a new territory of vacua that might give rise to the Standard Model. Also we may be able to see more nontrivial connections among different vacua, even not being shown connected in this paper. It would also be interesting to study these questions in non-prime orbifolds [48].

\section{Acknowledgments}

We are grateful to Michael Ratz, Soo-Jong Rey for discussions and Angel Uranga for a correspondence. K.S.C. is grateful to Theory Group of Hokkaido University for hospitality where this work is initiated. He also thanks to Theory Group of University of California, Irvine where this work is finished. K.S.C. is partly supported by grants NRF2015R1D1A1A01059940 and NRF-2018R1A2B2007163 of National Research Foundation of Korea. T.K. is partly supported by grant MEXT KAKENHI Grant Number JP17H05395.

Open Access. This article is distributed under the terms of the Creative Commons Attribution License (CC-BY 4.0), which permits any use, distribution and reproduction in any medium, provided the original author(s) and source are credited.

\section{References}

[1] E. Witten, Small instantons in string theory, Nucl. Phys. B 460 (1996) 541 [hep-th/9511030] [INSPIRE].

[2] M.J. Duff, R. Minasian and E. Witten, Evidence for heterotic/heterotic duality, Nucl. Phys. B 465 (1996) 413 [hep-th/9601036] [INSPIRE].

[3] O.J. Ganor and A. Hanany, Small E $E_{8}$ instantons and tensionless noncritical strings, Nucl. Phys. B 474 (1996) 122 [hep-th/9602120] [INSPIRE].

[4] D.R. Morrison and C. Vafa, Compactifications of F-theory on Calabi-Yau threefolds. 1, Nucl. Phys. B 473 (1996) 74 [hep-th/9602114] [INSPIRE].

[5] D.R. Morrison and C. Vafa, Compactifications of F-theory on Calabi-Yau threefolds. 2., Nucl. Phys. B 476 (1996) 437 [hep-th/9603161] [INSPIRE].

[6] M. Bershadsky, K.A. Intriligator, S. Kachru, D.R. Morrison, V. Sadov and C. Vafa, Geometric singularities and enhanced gauge symmetries, Nucl. Phys. B 481 (1996) 215 [hep-th/9605200] [INSPIRE].

[7] B. Haghighat, G. Lockhart and C. Vafa, Fusing E-strings to heterotic strings: $E+E \rightarrow H$, Phys. Rev. D 90 (2014) 126012 [arXiv:1406.0850] [INSPIRE].

[8] J. Kim, S. Kim, K. Lee, J. Park and C. Vafa, Elliptic Genus of E-strings, JHEP 09 (2017) 098 [arXiv : 1411.2324] [INSPIRE].

[9] K.-S. Choi and S.-J. Rey, E(lementary) Strings in Six-Dimensional Heterotic F-theory, JHEP 09 (2017) 092 [arXiv:1706.05353] [INSPIRE]. 
[10] K.-S. Choi and S.-J. Rey, Elliptic Genus, Anomaly Cancellation and Heterotic M-theory, arXiv: 1710.07627 [INSPIRE].

[11] L.J. Dixon, J.A. Harvey, C. Vafa and E. Witten, Strings on Orbifolds, Nucl. Phys. B 261 (1985) 678 [INSPIRE].

[12] L.J. Dixon, J.A. Harvey, C. Vafa and E. Witten, Strings on Orbifolds. 2., Nucl. Phys. B 274 (1986) 285 [INSPIRE].

[13] M. Berkooz, R.G. Leigh, J. Polchinski, J.H. Schwarz, N. Seiberg and E. Witten, Anomalies, dualities and topology of $D=6 N=1$ superstring vacua, Nucl. Phys. B 475 (1996) 115 [hep-th/9605184] [INSPIRE].

[14] J. Erler, Anomaly cancellation in six-dimensions, J. Math. Phys. 35 (1994) 1819 [hep-th/9304104] [INSPIRE].

[15] S. Kachru and C. Vafa, Exact results for $N=2$ compactifications of heterotic strings, Nucl. Phys. B 450 (1995) 69 [hep-th/9505105] [INSPIRE].

[16] G. Aldazabal, A. Font, L.E. Ibáñez and F. Quevedo, Chains of $N=2, D=4$ heterotic type-II duals, Nucl. Phys. B 461 (1996) 85 [hep-th/9510093] [INSPIRE].

[17] G. Aldazabal, A. Font, L.E. Ibáñez, A.M. Uranga and G. Violero, Nonperturbative heterotic $D=6, D=4, N=1$ orbifold vacua, Nucl. Phys. B 519 (1998) 239 [hep-th/9706158] [INSPIRE].

[18] K.-S. Choi and J.E. Kim, Z(2) orbifold compactification of heterotic string and 6-D SO(14) flavor unification model, Phys. Lett. B 552 (2003) 81 [hep-th/0206099] [INSPIRE].

[19] G. Honecker and M. Trapletti, Merging Heterotic Orbifolds and K3 Compactifications with Line Bundles, JHEP 01 (2007) 051 [hep-th/0612030] [INSPIRE].

[20] C. Lüdeling and F. Ruehle, F-theory duals of singular heterotic K3 models, Phys. Rev. D 91 (2015) 026010 [arXiv: 1405.2928] [INSPIRE].

[21] K.A. Intriligator, $R G$ fixed points in six-dimensions via branes at orbifold singularities, Nucl. Phys. B 496 (1997) 177 [hep-th/9702038] [INSPIRE].

[22] T. Eguchi, P.B. Gilkey and A.J. Hanson, Gravitation, Gauge Theories and Differential Geometry, Phys. Rept. 66 (1980) 213 [INSPIRE].

[23] P. Candelas, G.T. Horowitz, A. Strominger and E. Witten, Vacuum Configurations for Superstrings, Nucl. Phys. B 258 (1985) 46 [InSPIRE].

[24] K. Dasgupta, G. Rajesh and S. Sethi, M theory, orientifolds and G-flux, JHEP 08 (1999) 023 [hep-th/9908088] [INSPIRE].

[25] N. Seiberg and E. Witten, Comments on string dynamics in six-dimensions, Nucl. Phys. B 471 (1996) 121 [hep-th/9603003] [INSPIRE].

[26] A. Sagnotti, A Note on the Green-Schwarz mechanism in open string theories, Phys. Lett. B 294 (1992) 196 [hep-th/9210127] [INSPIRE].

[27] E. Witten, $\sigma$-models and the ADHM construction of instantons, J. Geom. Phys. 15 (1995) 215 [hep-th/9410052] [INSPIRE].

[28] P. Hořava and E. Witten, Heterotic and type-I string dynamics from eleven-dimensions, Nucl. Phys. B 460 (1996) 506 [hep-th/9510209] [INSPIRE]. 
[29] P. Hořava and E. Witten, Eleven-dimensional supergravity on a manifold with boundary, Nucl. Phys. B 475 (1996) 94 [hep-th/9603142] [INSPIRE].

[30] S. Groot Nibbelink, M. Trapletti and M. Walter, Resolutions of $C^{n} / Z_{n}$ Orbifolds, their U(1) Bundles and Applications to String Model Building, JHEP 03 (2007) 035 [hep-th/0701227] [INSPIRE].

[31] S. Groot Nibbelink, T.-W. Ha and M. Trapletti, Toric Resolutions of Heterotic Orbifolds, Phys. Rev. D 77 (2008) 026002 [arXiv:0707.1597] [InSPIRE].

[32] K.-S. Choi and J.E. Kim, Quarks and leptons from orbifolded superstring, Lect. Notes Phys. 696 (2006) 1 [INSPIRE].

[33] L.E. Ibáñez, J. Mas, H.-P. Nilles and F. Quevedo, Heterotic Strings in Symmetric and Asymmetric Orbifold Backgrounds, Nucl. Phys. B 301 (1988) 157 [InSPIRE].

[34] Y. Katsuki, Y. Kawamura, T. Kobayashi, N. Ohtsubo, Y. Ono and K. Tanioka, $Z(N)$ orbifold models, Nucl. Phys. B 341 (1990) 611 [INSPIRE].

[35] K.-S. Choi, S. Groot Nibbelink and M. Trapletti, Heterotic SO(32) model building in four dimensions, JHEP 12 (2004) 063 [hep-th/0410232] [INSPIRE].

[36] L.J. Dixon, Symmetry Breaking in String Theories via Orbifolds, Ph.D. Thesis, Princeton University (1986).

[37] K.-S. Choi, K. Hwang and J.E. Kim, Dynkin diagram strategy for orbifolding with Wilson lines, Nucl. Phys. B 662 (2003) 476 [hep-th/0304243] [INSPIRE].

[38] K.S. Narain, New Heterotic String Theories in Uncompactified Dimensions < 10, Phys. Lett. 169B (1986) 41 [INSPIRE].

[39] K.S. Narain, M.H. Sarmadi and E. Witten, A Note on Toroidal Compactification of Heterotic String Theory, Nucl. Phys. B 279 (1987) 369 [InSPIRE].

[40] K.-S. Choi, Unification in intersecting brane models, Phys. Rev. D 74 (2006) 066002 [hep-th/0603186] [INSPIRE].

[41] K.-S. Choi, Intersecting brane world from type-I compactification, Int. J. Mod. Phys. A 22 (2007) 3169 [hep-th/0610026] [INSPIRE].

[42] A.N. Schellekens and N.P. Warner, Anomalies and Modular Invariance in String Theory, Phys. Lett. B 177 (1986) 317 [inSPIRE].

[43] A.N. Schellekens and N.P. Warner, Anomaly Cancellation and Selfdual Lattices, Phys. Lett. B 181 (1986) 339 [INSPIRE].

[44] M. Berkooz and R.G. Leigh, A D = $4 N=1$ orbifold of type-I strings, Nucl. Phys. B 483 (1997) 187 [hep-th/9605049] [INSPIRE].

[45] E. Gorbatov, V.S. Kaplunovsky, J. Sonnenschein, S. Theisen and S. Yankielowicz, On heterotic orbifolds, M-theory and type-I-prime brane engineering, JHEP 05 (2002) 015 [hep-th/0108135] [INSPIRE].

[46] S. Förste, H.P. Nilles, P.K.S. Vaudrevange and A. Wingerter, Heterotic brane world, Phys. Rev. D 70 (2004) 106008 [hep-th/0406208] [INSPIRE].

[47] T. Kobayashi, S. Raby and R.-J. Zhang, Constructing 5-D orbifold grand unified theories from heterotic strings, Phys. Lett. B 593 (2004) 262 [hep-ph/0403065] [INSPIRE].

[48] K.S. Choi and T. Kobayashi, work in progress. 